\title{
Semi-Automated Optimization of the CHARMM36 Lipid Force Field to Include Explicit Treatment of Long-Range Dispersion
}

\author{
Yalun Yu, ${ }^{\mathrm{a}, \mathrm{b}}$ Andreas Krämer, ${ }^{\mathrm{b}}$ Richard M. Venable, ${ }^{\mathrm{b}}$ Andrew C. Simmonett, ${ }^{\mathrm{b}}$ Alexander D. MacKerell, Jr., ${ }^{\mathrm{c}}$ \\ Jeffery B. Klauda, ${ }^{* a, d}$ Richard W. Pastor, ${ }^{*, b}$ and Bernard R. Brooks ${ }^{*}$,

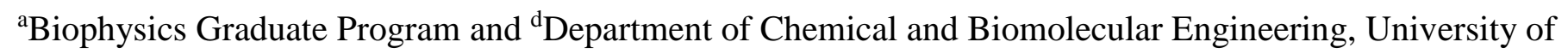 \\ Maryland, College Park, Maryland 20742, USA \\ baboratory of Computational Biology, National Heart, Lung and Blood Institute, National Institutes of Health, \\ Bethesda, MD 20892, USA
}

'Department of Pharmaceutical Sciences, School of Pharmacy, University of Maryland, Baltimore, Maryland 21201, USA

\begin{abstract}
The development of the CHARMM lipid force field (FF) can be traced back to the early 1990s with its current version denoted CHARMM36 (C36). The parametrization of C36 utilized high-level quantum mechanical data and free energy calculations of model compounds before parameters were manually adjusted to yield agreement with experimental properties of lipid bilayers. While such manual fine-tuning of FF parameters is based on intuition and trial-and-error, automated methods can identify beneficial modifications of the parameters via their sensitivities and thereby guide the optimization process. This paper introduces a semi-automated approach to reparametrize the CHARMM lipid FF with consistent inclusion of long-range dispersion through the LennardJones particle-mesh Ewald (LJ-PME) approach. The optimization method is based on thermodynamic reweighting with regularization with respect to the C36 set. Two independent optimizations with different topology restrictions are presented. Targets of the optimizations are primarily liquid crystalline phase properties of lipid bilayers and the compression isotherm of monolayers. Pair correlation functions between water and lipid functional groups in aqueous solution are also included to address headgroup hydration. While the physics of the reweighting strategy itself is well understood, applying it to heterogeneous, complex anisotropic systems poses additional challenges. These were overcome through careful selection of target properties and reweighting settings allowing for the successful incorporation of the explicit treatment of long-range dispersion, and we denote the newly optimized lipid force field as C36/LJ-PME. The current implementation of the optimization protocol will facilitate the future development of the CHARMM and related lipid force fields.
\end{abstract}




\section{Introduction}

Over the last decade, molecular dynamics (MD) simulations of membranes ${ }^{1-7}$ and membrane proteins ${ }^{8-12}$ have generated tremendous insight into biomolecular structures and processes. Because of their ability to generate atomistic details of molecular structure and kinetics, simulations have become a standard tool to guide, interpret, and complement experiments. The recent breakthroughs of MD simulations are mostly related with the larger length and time scales now accessible through modern computers, as well as force fields (FFs) that accurately reproduce structural bilayer properties under experimental conditions. ${ }^{13-17}$ The CHARMM36 (C36) set has been especially successful. It covers many important lipid types ${ }^{14,18-24}$ and is well-validated for various properties such as bilayer areas, compressibilities, spontaneous curvature, and bending constants. ${ }^{25}$ However, monolayer surface tensions from C36 substantially underestimate experiment, ${ }^{14}$ as expected from the lack of long-range dispersion. ${ }^{26-28}$ A solution to this is to include long-range LJ interactions explicitly through the LJ-PME method, ${ }^{27}$ however, the inclusion of this term requires additional parametrization of the FF due to substantial degradation in the reproduction of experimental observables.

A major challenge in lipid FF development is the complexity of the physics underlying the parametrization problem, and usually one can only focus on limited aspects of the system of interest under certain conditions (e.g., pressure, temperature, $\mathrm{pH}$, ionic strength). Furthermore, a rigorous description of certain properties may not even be possible with a particular class of FF. For example, additive FFs are deficient in regions where the dielectric response is dominated by electronic polarization so that the permeability of water in bilayers is not well described by C36. ${ }^{49}$ Consequently, FF developers must decide the important scientific targets of their FFs, leading to different parametrization strategies used by different communities. The development of the CHARMM lipid FF has historically utilized quantum mechanical (QM) calculations and experimental data of model compounds before selected parameters are manually adjusted to reproduce experimental properties of lipid bilayers. ${ }^{14,30}$ Such an approach is necessary as building a lipid FF solely based on small molecules that are representative of the various functional groups in a lipid but would neglect the interactions between these groups within a lipid and from neighboring lipids in the condensed phase. As a result, additional efforts are usually required to refine the parameters. For example, developers of the C36 lipid FF tested several methyl acetate (MAS) models before the final set was selected based on the accurate reproduction of bilayer experimental data. ${ }^{14,31}$ This procedure is consistent with the rest of the CHARMM additive FF allowing for application of the FF to complex, heterogeneous systems.

Both the optimization and validation of FFs require significant computational resources. MD simulations of lipid bilayers in the 1990 s were restricted to small systems and sub-nanosecond time scales, ${ }^{32-34}$ which greatly limited the development of reliable lipid FFs. This situation has significantly improved over the last two decades. With the increase of computer power and a variety of MD engines supporting parallel computing and/or graphics 
processing unit (GPU)-acceleration, ${ }^{35-38}$ a microsecond all-atom simulation of a thousand lipids is well within capabilities of most computer clusters. However, the atomistic simulation methods still suffer from the fact that the range of time and length scales by which different processes occur in membranes is vast. ${ }^{39}$ For a simple calculation of bilayer surface area, the uncertainty from a simulation on the $100 \mathrm{~ns}$ timescale is typically less than $1 \% .^{25,40,41}$ This error is acceptable in most circumstances but complicates optimization with noisy parameter sensitivities computed through finite differences. ${ }^{42,43}$ Furthermore, simply obtaining the surface area within the experimental error (which can be larger than 1\%) does not assure the accuracy of other properties. Finally, lipid FFs are frequently subjected to adjustments to study new experimental results and utilize simulation approaches, so efficient parametrization methods are essential.

To address these problems, physics-informed methods can be used to identify beneficial modifications of the parameters via reliable sensitivity evaluations and thereby guide the optimization process in a more efficient way. ${ }^{44-46}$ This manuscript introduces a semi-automated optimization approach for the CHARMM lipid FF. While both manual and automated FF parametrization have a long history, those two approaches have developed mostly independently. Fully automated approaches were long limited to low-dimensional parameter spaces ${ }^{44,47-49}$ and have only recently been leveraged to parametrize full FFs in the context of the OpenForceField Initiative. ${ }^{50,51}$ Automated optimization procedures for united-atom lipid FFs have been presented in Refs ${ }^{52,53}$. Such automated workflows have been developed for a gamut of numerical optimization algorithms, including gradient-based methods $^{44,48,49}$ as well as global optimization methods based on evolutionary, ${ }^{42}$ simplex, ${ }^{47,53}$ and metamodelbased optimization. ${ }^{42,46}$ However, FF optimization problems are often underdetermined, which can easily lead to unreasonable parameters that are generally not transferrable to simulations setups outside the training set. Therefore, the most used FFs to this day are still based on manual development.

The present work combines automated and manual approaches. While chemical intuition and consistency with the CHARMM FF are encoded in the form of restraints and suitable weights, new iterations of the FF are generated by an automated strategy based on thermodynamic reweighting. This approach allows the incorporation of intricacies specific to the lipid FF. While the primary goal in this paper is to incorporate the Lennard-Jones particle-mesh Ewald (LJ-PME) method ${ }^{54}$ into the CHARMM36 lipid FF, the general methodology is transferable to other modifications and other lipid FFs. This paper describes the method and presents the training set, using phosphatidylcholine (PC) lipids as benchmarks. The validation including additional observables and lipid types is presented in the second part of this study "CHARMM36 Lipid Force Field with Explicit Treatment of LongRange Dispersion: Parametrization and Validation for PE, PG, and ether lipids” by Yu et al. [citation to paper II] (henceforth denoted Paper II). 


\section{Methods}

2.1. Parameters to Optimize. Our previous study demonstrated that the C36 lipid FF reproduces experimental observables very well for linear alkanes when the long-range LJ interactions are included, ${ }^{27}$ so that it is reasonable to maintain the alkane parameters and only modify the head group parameters for the reparametrization. The next question concerns which part of the head group should be adjusted. To explore the outcomes of different choices, we performed two separate optimizations. In the first, denoted Global, all the nonbonded parameters of the head group along with selected torsions were subject to change. In the other, denoted Linkage, only the nonbond parameters of the glycerol and ester groups were optimized to maximize consistency with the rest of the CHARMM FF; e.g., the phosphate nonbond parameters would remain the same for lipids, proteins, and DNA. Table S1 presents the nonbond parameters explicitly optimized in both the Global and Linkage sets.

2.2. General Optimization Procedure. The optimization strategy (Figure 1), FFLiP (Force Field of Lipid Parametrization), was inspired by earlier gradient-based parametrization strategies, ${ }^{48,} 49$ especially the ForceBalance protocol. ${ }^{44}$ Our starting point was the C36 set, which has been parametrized targeting reliable experimental data and high-level QM calculations. Lipid-containing systems were initially simulated with this parameter set. Upon completion of the simulations, equilibrium properties $\boldsymbol{f}^{\text {sim }}$ and potential energies $U$ were calculated for each trajectory frame. The same trajectories were used for potential energy re-calculations in OpenMM 7.4.0 ${ }^{38}$ with the perturbed parameter sets. Parameter sensitivities (gradients) of the equilibrium properties were estimated through thermodynamic reweighting 55

$$
\boldsymbol{S}_{\text {prop }}(\boldsymbol{\lambda}, \boldsymbol{\delta} \boldsymbol{\lambda})=\left\langle\boldsymbol{f}^{s i m}\right\rangle_{\lambda+\delta \lambda}-\left\langle\boldsymbol{f}^{\operatorname{sim}}\right\rangle_{\lambda}=\frac{\left\langle\boldsymbol{f}^{\operatorname{sim}} e^{-\beta\left(U_{\lambda+\delta \lambda}-U_{\lambda}\right)}\right\rangle_{\lambda}}{\left\langle e^{-\beta\left(U_{\lambda+\delta \lambda}-U_{\lambda}\right)}\right\rangle_{\lambda}}-\left\langle\boldsymbol{f}^{s i m}\right\rangle_{\lambda}
$$

where $\boldsymbol{S}_{\text {prop }}$ is the sensitivity matrix of properties, $\lambda$ is the original parameter set and $\delta \lambda$ is the perturbation of that parameter set. $\langle\cdot\rangle_{\lambda}$ denotes the average over trajectory, which is also the ensemble average of parameter set $\lambda$, approximately. $\boldsymbol{S}_{\text {prop }}$ was calculated in a parallel fashion because different parameters can be calculated independently. In the next step, the prediction for the best new set of nonbonded parameters $\boldsymbol{P}$ is given by solving Eq. (2) in a least-square manner

$$
\min !\|\boldsymbol{W}(\boldsymbol{S} \cdot \boldsymbol{P}-\boldsymbol{F})\|
$$




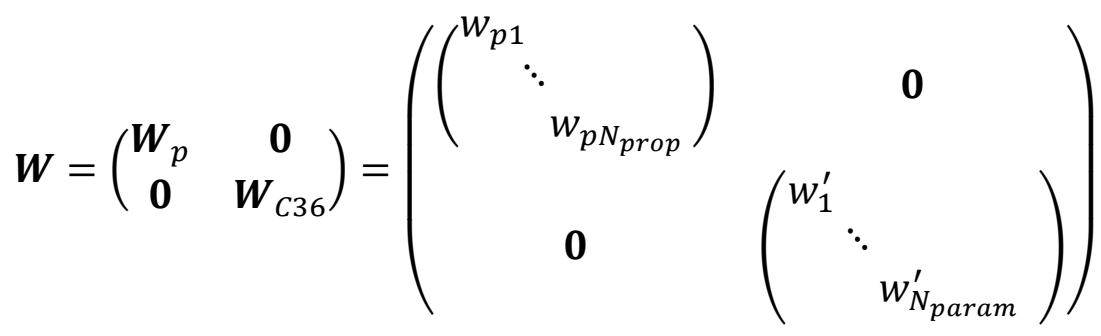

$$
\begin{aligned}
& \left.\boldsymbol{S}=\left(\begin{array}{c}
\boldsymbol{S}_{\text {prop }} \\
\boldsymbol{S}_{\text {param }}
\end{array}\right)=\left(\begin{array}{ccc}
S_{1,1} & \cdots & s_{1, N_{\text {param }}} \\
\vdots & \ddots & \vdots \\
S_{N_{\text {prop }}, 1} & \cdots & s_{N_{\text {prop }}, N_{\text {param }}}
\end{array}\right)\right) \\
& \boldsymbol{F}=\left(\begin{array}{c}
\boldsymbol{F}_{\text {prop }} \\
\boldsymbol{F}_{C 36}
\end{array}\right)=\left(\begin{array}{c}
\boldsymbol{f}^{e x p}-\left\langle\boldsymbol{f}^{s i m}\right\rangle_{\lambda} \\
0 \\
\vdots \\
0
\end{array}\right)
\end{aligned}
$$

where $\boldsymbol{W}$ is a diagonal weight matrix composed of apparent weights for the target properties $\left(\boldsymbol{W}_{p}\right)$ and weights used for regularization $\left(\boldsymbol{W}_{\mathrm{C} 36}\right) ; \boldsymbol{S}$ is the complete sensitivity matrix, which consists of the sensitivity matrix of the target properties and an identity matrix of size $N_{\text {param }}$ (the total number of parameters); $\boldsymbol{F}$ is the target vector, which contains the deviations of simulated results from the experiments and $N_{\text {param }}$ zeros to restrain the parameter set to C36. The least-square problem was solved by the "linalg.lstsq" method in NumPy, which minimizes the Euclidean 2-norm $\|\boldsymbol{W}(\boldsymbol{S} \cdot \boldsymbol{P}-\boldsymbol{F})\|$ when the equation is overdetermined ${ }^{56}$ While the number of parameters is not necessarily less than the experimental targets (which means underdetermination of the problem in the language of linear algebra), we can avoid this situation by adding parameter restraints (which correspond to $\boldsymbol{F}_{C 36}$ in the $\boldsymbol{F}$ matrix) as additional equations to make sure the number of equations are more than the number of parameters. These additional equations all have the simple form of $\Delta P_{i}=0\left(P_{i}\right.$ stands for the $i^{\text {th }}$ parameter $)$ and correlate with the $\boldsymbol{W}_{\mathrm{C} 36}$ part in the weight matrix and the identity matrix $\boldsymbol{I}_{N_{\text {param }}}$ in the sensitivity matrix. They serve as restraints and ensure that Eq. (2) is overdetermined, and a least-square solution is available. Dihedral parameters associated with the changed nonbonded parameters were adjusted at the end of each optimization cycle through reweighting or direct fitting (see Section 2.4). A new set of simulations was subsequently run to test the new parameters. If the results were satisfactory, the optimization would be terminated. If not, the cycle would continue until optimal parameters were obtained. 


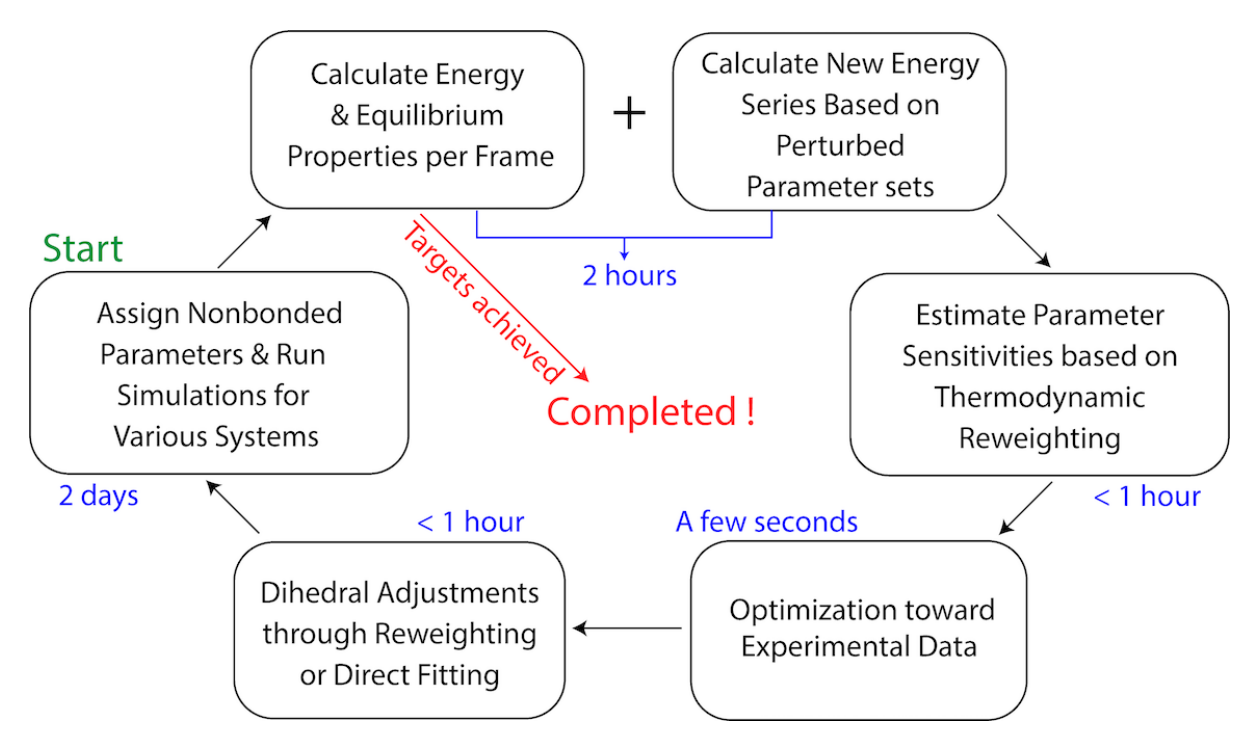

Figure 1. The optimization procedure and approximate time per step.

2.3. Training Set. The common goal of parametrizing a lipid FF is to find the parameters that reproduce experimentally measured properties. As a result, the quality of a lipid FF is heavily influenced by the availability of experimental data and the quality of the fitting process. For lipids, there are abundant experimental data available including surface area per lipid of single component bilayer, monolayer isotherms, NMR deuterium order parameter, area compressibility, bending constant, NMR relaxation time, and lipid diffusion constant. These experiments provide valuable information regarding the structures, mechanical properties, and dynamics of lipid bilayers and monolayers. The atom-pair-specific radial distribution functions (RDFs) between the phosphate group/ester linkage and water molecules determined recently by Mclain et al ${ }^{57,58}$ provide insights to the hydration of the head groups. However, when choosing the training set, not all of these experiments can be used, especially for dynamic properties, since the reweighting strategy is most efficient when applied to thermodynamic properties that can be computed for individual configurations. In principle, dynamic properties can be added to the training set if the parameter space is sufficiently explored in advance so that a model can be built to predict the results based on the input parameters. The parameter space here is too large to explore and develop such a model, so dynamic properties were excluded from the training set, but were tested in paper II as part of validation. The training targets for the two optimizations are listed in Table 1. The current parametrizations focus on PC lipids, which include DPPC, 1,2-dimyristoyl-sn-glycero-3-phosphorylcholine (DMPC), 1-palmitoyl-2-oleoyl-snglycero-3-phosphocholine (POPC) and 1,2-dipropionyl-sn-glycero-3-phosphocholine ( $\left.\mathrm{C}_{3}-\mathrm{PC}\right)$. In the Linkage optimization, the RMSD of $\mathrm{C}_{3}$-PC RDF was used instead of the maximum and minimum used in the Global optimization, partly due to the fixing of the phosphate parameters. 
Table 1. Training target data, along with their scaling factors and weight factors. Properties cover surface area per lipid $\left(A_{l}\right)$, deuterium order parameters $\left(S_{\mathrm{CD}}\right)$, overall bilayer thickness $\left(D_{\mathrm{B}}\right)$, and radial distribution function (RDF). $S_{\mathrm{CD}}$ (tail) refer to chain order parameters from the $4^{\text {th }}$ carbon in each tail with available experimental data. The simulation systems used to extract each property are shown in the second column with temperature (the surface tension per leaflet is shown in parenthesis if nonzero), and the hydration number for each system is shown in the third column $\left(N_{\text {water/lipid }}\right)$. Scaling and weight factors are defined Section 2.5.

\begin{tabular}{|c|c|c|c|c|c|c|}
\hline property & system, temperature $(\mathrm{K})$ & $N_{\text {waterlipid }}$ & target value & $\begin{array}{l}\text { scaling } \\
\text { factor }\end{array}$ & $\begin{array}{l}\text { weight factor } \\
\text { - Global }\end{array}$ & $\begin{array}{l}\text { weight factor } \\
\text { - Linkage }\end{array}$ \\
\hline$A_{l}$ & \multirow{5}{*}{ DPPC Bilayer, 323.15} & \multirow{5}{*}{30.4} & $63.1\left(\AA^{2}\right)^{59}$ & $60 \AA^{2}$ & 20 & 20 \\
\hline$S_{\mathrm{CD}}($ tail $)$ & & & $\operatorname{ref}^{60,61}$ & 0.15 & $0.05 \times 11$ & $0.05 \times 11$ \\
\hline$S_{\mathrm{CD}}$ (head group) & & & $\operatorname{ref}^{62}$ & 0.15 & $0.5 \times 11$ & $0.5 \times 11$ \\
\hline$D_{B}$ & & & $39.0(\AA)^{59}$ & $40 \AA$ & 5 & 5 \\
\hline$\Delta A_{l}(\mathrm{DMPC}-\mathrm{DPPC})$ & & & $2.5\left(\AA^{2}\right)^{59,63}$ & $2 \AA$ & 1 & 1 \\
\hline$A_{l}$ & \multirow[t]{2}{*}{ DMPC Bilayer, 303.15} & \multirow[t]{2}{*}{25.7} & $60.6\left(\AA^{2}\right)^{63}$ & $60 \AA^{2}$ & 10 & 10 \\
\hline$D_{B}$ & & & $36.7(\AA)^{59}$ & $40 \AA$ & 5 & 10 \\
\hline$A_{l}$ & \multirow{2}{*}{ DPPC Bilayer, 333.15} & \multirow{2}{*}{30.4} & $65.0\left(\AA^{2}\right)^{59}$ & $60 \AA^{2}$ & 10 & 10 \\
\hline$D_{B}$ & & & $38.1(\AA)^{59}$ & $40 \AA$ & 5 & 5 \\
\hline$A_{l}$ & \multirow{2}{*}{ DMPC Bilayer, 303.15} & \multirow{2}{*}{25.7} & $60.6\left(\AA^{2}\right)^{63}$ & $60 \AA^{2}$ & 10 & 10 \\
\hline$D_{B}$ & & & $36.7(\AA)^{59}$ & $40 \AA$ & 5 & 10 \\
\hline$A_{l}$ & POPC Bilayer, 303.15 & 31.1 & $64.3\left(\AA^{2}\right)^{59}$ & $60 \AA^{2}$ & 5 & 5 \\
\hline$A_{l}$ & $\begin{array}{l}\text { DPPC Monolayer (18 } \\
\text { dyn/cm), } 321\end{array}$ & 30.4 & $54\left(\AA^{2}\right)^{64}$ & $60 \AA^{2}$ & 5 & 5 \\
\hline$A_{l}$ & $\begin{array}{l}\text { DPPC Monolayer (40 } \\
\text { dyn/cm), } 321\end{array}$ & 30.4 & $64\left(\AA^{2}\right)^{64}$ & $60 \AA^{2}$ & 5 & 5 \\
\hline$A_{l}$ & $\begin{array}{l}\text { DPPC Monolayer (55 } \\
\text { dyn/cm), } 321\end{array}$ & 30.4 & $80\left(\AA^{2}\right)^{64}$ & $60 \AA^{2}$ & 5 & 5 \\
\hline $\begin{array}{l}\text { RDF (water atom } \sim \text { lipid } \\
\text { atom) Maximum }\end{array}$ & \multirow{3}{*}{$\mathrm{C}_{3}$-PC in solution, 298.15} & \multirow{3}{*}{250} & \multirow{3}{*}{$\operatorname{ref}^{57}$} & 1 & $0.75 \times 10$ & 0 \\
\hline $\begin{array}{l}\text { RDF (water atom } \sim \text { lipid } \\
\text { atom) Minimum }\end{array}$ & & & & 0.5 & $0.75 \times 6$ & 0 \\
\hline $\begin{array}{l}\text { RMSD of RDF from } 0.2 \text { to } \\
0.6 \mathrm{~nm}\end{array}$ & & & & 1 & 0 & 1.5 \\
\hline \multirow{3}{*}{$\begin{array}{l}K_{A} \text { of DPPC Bilayer at } \\
323.15 \mathrm{~K}\end{array}$} & $\begin{array}{l}\text { DPPC Bilayer (5 } \\
\text { dyn/cm), } 323.15\end{array}$ & 30.4 & \multirow{2}{*}{$\begin{array}{l}231 \\
(\mathrm{dyn} / \mathrm{cm})^{65}\end{array}$} & \multirow{2}{*}{$\begin{array}{l}200 \\
\mathrm{dyn} / \mathrm{cm}\end{array}$} & \multirow{2}{*}{0.75} & \multirow{2}{*}{0.75} \\
\hline & $\begin{array}{l}\text { DPPC Bilayer (-5 } \\
\text { dyn/cm), } 323.15\end{array}$ & 30.4 & & & & \\
\hline & DPPC Bilayer, 323.15 & 30.4 & $0.19\left(\AA^{2} / K\right)^{59}$ & $0.2 \AA^{2} / \mathrm{K}$ & 1 & 1 \\
\hline
\end{tabular}




\begin{tabular}{|l|l|l|l|l|l|l|}
\hline $\begin{array}{l}\text { DPPC Bilayer Isobaric } \\
\text { Expansion }\end{array}$ & DPPC Bilayer, 333.15 & 30.4 & & & & \\
\hline
\end{tabular}

2.4. Dihedral Fitting. Dihedral parameters play a critical role in bond orientations and dynamics at the atomic level. The C36 lipid FF yields excellent agreement with the experimental deuterium order parameters in the head group region, ${ }^{1,66}$ which can be credited to the extensive QM calculations of model compounds representative of the head group and linkage region. Although fitting to the same set of QM data is a reasonable strategy to take for C36/LJ-PME, running the QM calculations has the added difficulty because one needs to find the conformations that are representative of the lipid in a biological environment. In fact, the C36 dihedrals are good targets for several reasons. First, it is easy to hold the model compounds in fixed conformations when comparing the fitted set and C36. Second, the potential energy scans are easier to obtain for C36 compared to the QM calculations. Last, fitting to C36 is expected to reproduce the order parameters which are already in good agreement with experiments. When fitting to $\mathrm{C} 36$, two methods were used. The first is fitting to the potential energy scan (PES) of a dihedral using a model compound. The second is fitting through thermodynamic reweighting (Eq. 1). In the first method, all dihedrals but the scanned dihedral were unconstrained, while the scanned dihedral was restrained using a force constant of $10^{3} \mathrm{kcal} / \mathrm{mol}$ during the energy minimization consisting of 200 steepest decent steps and 1000 adopted basis Newton-Raphson steps in CHARMM. In the second method, a 10-ns trajectory block from a DPPC bilayer simulation was used to calculate the torsion angles and the corresponding energy series. In addition, a trajectory sampled by C36 was used to obtain the reference torsion distributions. The dihedral parameters were changed iteratively using the Broyden-Fletcher-Goldfarb-Shanno (BFGS) algorithm implemented in scipy ${ }^{56}$ until the convergence criteria were met. Table 2 shows the dihedrals fitted in each optimization cycle and the corresponding fitting protocols. The choice of fitting protocol was conditioned on the availability of QM conformational energies from the original publication of C36. ${ }^{14} \mathrm{~A}$ Monte Carlo simulated annealing approach ${ }^{67}$ was used for fitting to the QM/C36 PES. The results for dihedral fittings are discussed in Section 3.

Table 2. Dihedrals fitted in each optimization cycle and the corresponding fitting protocols. Atom names and model compounds are shown in Figure 2. CHARMM atom types included in parenthesis for each dihedral. Symbols for the dihedrals are consistent with C36 and are used henceforth.

\begin{tabular}{|c|c|c|c|}
\hline Dihedral & Symbol & Fitting Protocol & Model Compound \\
\cline { 1 - 2 } O11-C1-C2-C3 (OSLP-CTL2-CTL1-CTL2) & $\theta_{1}$ & Reweighting to C36 & None \\
\cline { 1 - 2 } C2-C3-O31-C31 (CTL1-CTL2-OSL-CL) & $\gamma_{1}$ & Torsion Distribution & \\
\cline { 1 - 2 } C1-C2-O21-C21(CTL2-CTL1-OSL-CL) & $\beta_{1}$ & & \\
\hline
\end{tabular}




\begin{tabular}{|c|c|c|c|}
\hline O11-C1-C2-O21 (OSLP-CTL2-CTL1-OSL) & $\theta_{2}$ & \multirow{2}{*}{$\begin{array}{c}\text { in DPPC Bilayer } \\
\text { Simulation }\end{array}$} & \\
\hline O21-C2-C3-O31 (OSL-CTL1-CTL2-OSL) & $\theta_{4}$ & & \\
\hline P-O12-C11-C12 (PL-OSLP-CTL2-CTL2) & $\alpha_{4}$ & \multirow{3}{*}{$\begin{array}{l}\text { Least Squares Fit to } \\
\text { C36 Potential Energy } \\
\text { Scan }\end{array}$} & PMP \\
\hline P-O11-C1-C2 (PL-OSLP-CTL2-CTL1) & $\alpha_{1}$ & & PMP \\
\hline O31-C31-C32-C33 (OSL-CL-CTL2-CTL2) & $\gamma_{3}$ & & EGLY \\
\hline $\begin{array}{c}\text { C31-C32-C33-C34/C21-C22-C23-C24 } \\
\text { (CL-CTL2-CTL2-CTL2) }\end{array}$ & $\beta_{4} / \gamma_{4}$ & $\begin{array}{l}\text { Least Squares Fit to } \\
\text { QM Potential Energy } \\
\text { Scan }\end{array}$ & $\mathrm{IPB} / \mathrm{PB}$ \\
\hline
\end{tabular}

DPPC

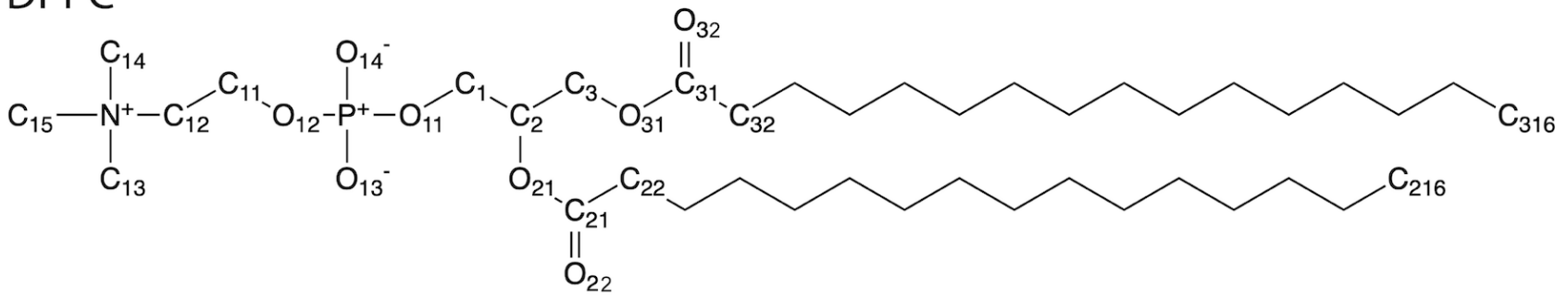

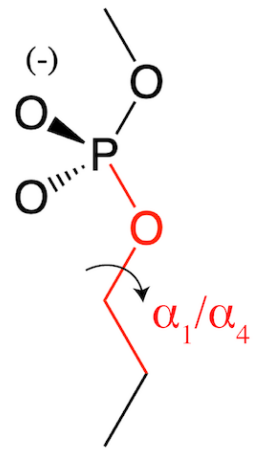

PMP (PGHG)

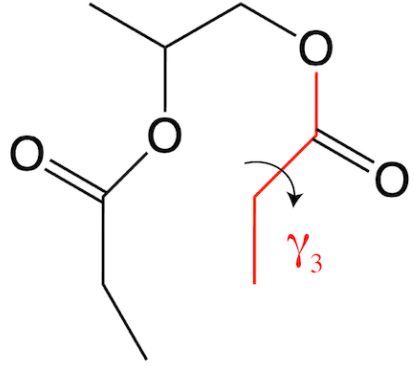

EGLY (GH2F)

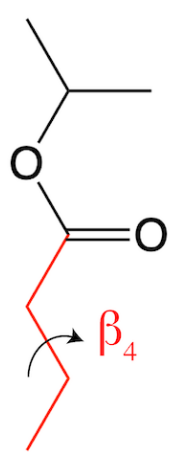

IPB (MEEF)

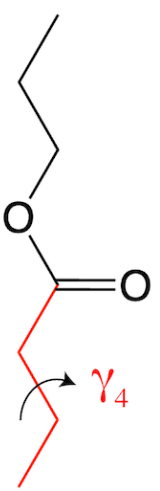

PB (ACTF)

Figure 2. Chemical structure of DPPC and model compounds used for dihedral fitting: propylmethyphosphate (PMP), an esterfied glycerol-phosphate analogue (EGLY). isopropylbutyrate (IPB), and propylbutyrate (PB). The CHARMM residue names shown in parenthesis. See Figure S1 for full atom notation including hydrogens.

2.5. Parameter Perturbation. The potential energy in the CHARMM FF is

$$
\begin{array}{r}
V(\hat{R})=\sum_{\text {bonds }} K_{b}\left(b-b_{0}\right)^{2}+\sum_{\text {angles }} K_{\theta}\left(\theta-\theta_{0}\right)^{2}+\sum_{\text {dihedrals }} \sum_{j} K_{\varphi, j}\left(1+\cos \left(n_{j} \varphi-\delta_{j}\right)\right) \\
+\sum_{\text {nonbonded pairs } i, j} \varepsilon_{i j}\left[\left(\frac{R_{\min , i j}}{r_{i j}}\right)^{12}-\left(\frac{R_{\min , i j}}{r_{i j}}\right)^{6}\right]+\sum_{\text {nonbonded pairs } i, j} \frac{q_{i} q_{j}}{\varepsilon_{D} r_{i j}}
\end{array}
$$


where the terms have their usual meanings. The bond and angle terms were left unchanged from C36. As described in Section 2.1, thermodynamic reweighting allows fast evaluations of the parameter sensitivities. However, such evaluations require sufficient conformational overlap between the original and perturbed states for accurate prediction; i.e., the perturbation $\boldsymbol{\delta} \boldsymbol{\lambda}$ in eq 1 must be sufficiently small to ensure this criterion is met. A detailed investigation of the magnitude of perturbations is presented in Section 3. Table 3 lists the perturbations used for all parameter types. The perturbations used for partial charges are $10^{-3} \mathrm{e}$. Percentage perturbations were used for the LJ parameters, since they differ substantially among different atom types. When perturbing the partial charge of an atom, the charge re-distributed to selected neighboring atoms to ensure a fixed net charge. The rules for charge re-distribution are shown in Table S2, which takes the CHARMM definition of integer charge groups into consideration so that the re-distribution was always within a charge group. When there were $N$ equivalent atom sites, for example, $\mathrm{O} 22$ and $\mathrm{O} 32$, perturbations to them occurred at the same time but the perturbation size for each was scaled by $1 / N$ to limit the conformational change corresponding to these perturbations.

Table 3. Perturbation sizes used for partial charge, $\varepsilon$ and $\mathrm{R}_{\min } / 2$ during optimization.

\begin{tabular}{|l|l|}
\hline Parameter Type & Perturbation Size \\
\hline$q$ & $10^{-3} \mathrm{e}$ \\
\hline$\varepsilon$ & $0.1 \%$ of the parameter in the last optimization step \\
\hline$R_{\min } / 2$ & $0.1 \%$ of the parameter in the last optimization step \\
\hline
\end{tabular}

2.5. Regularization. As shown in Eq. (2), the optimal parameter set $\boldsymbol{P}$ is given by solving a linear equation which used the sensitivity matrix and a weight matrix. The weight matrix consisted of two parts. The $i^{\text {th }}$ component of the first part, $\boldsymbol{w}_{p i}$, was set to be the weight factor of property $i$ divided by the scaling factor of property $i$ (see Table 1 for values). Scaling factors were used to make sure all training targets were at the same order of magnitude and weight factors were used to adjust the importance of the training targets. While the user can set the weights for the training targets (properties) according to their significances, the second part of the weight matrix, $\boldsymbol{W}_{C 36}$, is even more important because it determines how far the parameters can shift from the original C36 set. Such restraints, called regularizations, are usually applied to avoid overshooting of parameters during the fitting process. Incorporating the initial parameters into the target vector is equivalent to adding a harmonic restraint as in the L2 regularization used in ForceBalance. ${ }^{44}, 52$ To construct a meaningful $\boldsymbol{W}_{C 36}$, the relative magnitude of this submatrix (compared to $\boldsymbol{W}_{p}$ ) should be determined to assure that the change of the parameter set in each optimization step is suitably small. Moreover, the relative weights for charges and LJ parameters should be balanced properly, so that all parameters change in a physically meaningful way. The default weight factors for different parameter types in each optimization cycle can be found in Table S3. Apart from these, we used the 
standard deviations of parameter sensitivity estimated from three independent trajectory blocks to modify the default weight factors in each optimization step.

To justify this modification, consider a property that is determined by only a subset of the system's coordinates, $\boldsymbol{r}_{\mathbf{1}}$. Let $\boldsymbol{r}$ denote the full coordinate set, and $\boldsymbol{r}_{\mathbf{2}}$ those coordinates not belonging to $\boldsymbol{r}_{\mathbf{1}}$. The potential energy function, $U(\boldsymbol{r})$, can be decomposed as:

$$
U(\boldsymbol{r})=U\left(\boldsymbol{r}_{1}\right)+U\left(\boldsymbol{r}_{2}\right)+U\left(\boldsymbol{r}_{1}, \boldsymbol{r}_{2}\right)=U\left(\boldsymbol{r}_{1}\right)+U^{\prime}
$$

where $U\left(\boldsymbol{r}_{\mathbf{1}}\right)$ and $U\left(\boldsymbol{r}_{\mathbf{2}}\right)$ are contributions from subset $\boldsymbol{r}_{\mathbf{1}}$ and $\boldsymbol{r}_{\mathbf{2}}$, respectively, and $U\left(\boldsymbol{r}_{\mathbf{1}}, \boldsymbol{r}_{\mathbf{2}}\right)$ is the contribution from the cross-interactions between the two subsets. With this, an element of the sensitivity matrix in Eq. (1) can be reformatted accordingly as:

$$
s(\lambda, \delta \lambda)=\left\langle f^{\operatorname{sim}}\right\rangle_{\lambda+\delta \lambda}-\left\langle f^{\operatorname{sim}}\right\rangle_{\lambda}=\frac{\left\langle f^{\operatorname{sim}} e^{-\beta\left[U_{\lambda+\delta \lambda}\left(\boldsymbol{r}_{\mathbf{1}}\right)+U_{\lambda+\delta \lambda}^{\prime}-U_{\lambda}\left(\boldsymbol{r}_{\mathbf{1}}\right)-U_{\lambda}^{\prime}\right]}\right\rangle_{\lambda}}{\left\langle e^{-\beta\left[U_{\lambda+\delta \lambda}\left(\boldsymbol{r}_{\mathbf{1}}\right)+U_{\lambda+\delta \lambda}^{\prime}-U_{\lambda}\left(\boldsymbol{r}_{\mathbf{1}}\right)-U_{\lambda}^{\prime}\right]}\right\rangle_{\lambda}}-\left\langle f^{\operatorname{sim}}\right\rangle_{\lambda}
$$

Here, we use normal fonts for $s, f^{\operatorname{sim}}$ and $\lambda$ instead of bold to indicate that we only focus on one particular property and only one parameter. Suppose that the subset $\boldsymbol{r}_{\mathbf{1}}$ and $\boldsymbol{r}_{\mathbf{2}}$ can be chosen in a way such that $U\left(\boldsymbol{r}_{\mathbf{1}}\right)$ is strongly coupled with the property $f^{\text {sim }}$ while $U^{\prime}$ is only weakly coupled or is uncoupled. In such a case, if parameter $\lambda$ 's influence on $U\left(\boldsymbol{r}_{\mathbf{1}}\right)$ is substantial, then the property $s$ will be dominated by $\lambda$ as they are strongly coupled with each other through $U\left(\boldsymbol{r}_{1}\right)$. As a result, the gradient of $f^{s i m}$ in the direction of $\lambda$ estimated by the reweighting would be meaningful. However, if $U\left(\boldsymbol{r}_{\mathbf{1}}\right)$ is not substantially influenced by $\lambda$, or if a change in $\lambda$ leads to more change in $U^{\prime}$ rather than $U\left(\boldsymbol{r}_{\mathbf{1}}\right)$, the quality of the reweighting will be impaired since $U^{\prime}$ will act as a noise to the total potential energy. Although this noise can be reduced by extending the simulation to acquire enough sampling, it is not practical for our membrane systems (which contain more than $10^{4}$ atoms). An alternative is to set customized weights for parameters according to the standard deviations of the sensitivities estimated from trajectory blocks. Specifically, a candidate $w_{\lambda}$ for parameter $\lambda$ is calculated through

$$
w_{\lambda}=S_{b} * \frac{\sum_{\text {prop }}\left(w_{\text {prop }} \cdot \operatorname{STD}\left(s_{\text {prop }}\right)\right)}{\sum_{\text {prop }}\left(w_{\text {prop }}\right)}
$$

where $s_{\text {prop }}$ is the sensitivity for a particular property, $w_{\text {prop }}$ is the effective weight (weight factor/scaling factor) for the property, which is also used in $\boldsymbol{W}_{\boldsymbol{p}}$. STD means standard deviation, and $S_{\mathrm{b}}$ is a scaling factor used to 
balance $\boldsymbol{W}_{\boldsymbol{p}}$ and $\boldsymbol{W}_{C 36}$. The unit of $w_{\lambda}$ is $1 / \mathrm{e}$ for charges and $1 /$ percentage for $\varepsilon$ and $R_{\min } / 2$. During the optimization, $w_{\lambda}$ was used if it was greater than the default weight for the parameter type of $\lambda$, otherwise the default was kept. Moreover, if the calculated $w_{\lambda}$ was larger than a preset upper bound, the parameter would not be allowed to change in the optimization step. Consequently, parameter changes leading to large uncertainties were avoided or at least reduced in a property-dependent manner. Therefore, confidence was increased for the more important properties. $S_{\mathrm{b}}$ used in each optimization cycle can be found in Table S4, while the upper bounds were determined to be 20/e for charge or 20/percentage for $\varepsilon$ and $R_{\min } / 2$. Much higher $S_{\mathrm{b}}$ and default weighting factors (Table S3) were used for the last (third) optimization cycle in the Global optimization, because the simulated properties after the second cycle in that optimization were close to their target values so that harder restraints could be applied.

2.6. Computational Details. Membrane coordinates were obtained from the CHARMM-GUI ${ }^{68}$ Membrane $^{2}$ Builder ${ }^{69,70}$ and minimized/equilibrated following the standard six-step procedure offered by CHARMM-GUI. The $\mathrm{C}_{3}$ - $\mathrm{PC}$ residue was created by modifying DMPC, removing fatty acid chain $\mathrm{C} 4: \mathrm{C} 14$ and attached $\mathrm{H}$ atoms, and converting C3 from methylene to methyl; no new or modified parameters were required. A single molecule was built from the internal coordinates of the residue definition, and then subjected to 80 ns of vacuum SelfGuided Langevin Dynamics (SGLD), ${ }^{71}$ with a collision frequency of 1/ps and a dielectric constant of 80 to screen the charges. Nine configurations were randomly selected from the vacuum trajectory and placed on a grid with one molecule at the origin, and the other eight translated to the corners of a $20 \AA$ cube centered on the origin, and randomly rotated. Water coordinates from an equilibrated cube with a $43 \AA$ edge were read, and waters closer than $2.3 \AA$ to any $\mathrm{C}_{3}$-PC molecule were deleted. The system was relaxed with 50 steps of steepest descent minimization, followed by 1000 steps of the adopted-basis Newton-Raphson minimization. 36 lipids per leaflet (72 in total) are used for all membrane systems and the hydration number for each system is shown in Table 1.

In each optimization cycle, membrane systems were simulated for 200 ns with the exception being the two NP $\gamma$ T simulations of DPPC used to calculate the compressibility modulus, which were simulated for $300 \mathrm{~ns}$. The first $50 \mathrm{~ns}$ (for NPT simulations) or $60 \mathrm{~ns}$ (for NP $\gamma \mathrm{T}$ simulations) was discarded to avoid the unequilibrated part of the trajectory. The block sizes used for the standard deviation calculations of sensitivity were $50 \mathrm{~ns}$ and $80 \mathrm{~ns}$, respectively. The $\mathrm{C}_{3}-\mathrm{PC}$ system was simulated for $100 \mathrm{~ns}$ (first $10 \mathrm{~ns}$ discarded) in each optimization cycle and $30 \mathrm{~ns}$ blocks were used for the standard deviation calculation. For all systems, the modified TIP3P water model ${ }^{72,}$ ${ }^{73}$ was used to keep consistency with other parts of the force field. All the simulations were run in OpenMM 7.4.0 using the Langevin Integrator with a timestep of 2 fs. Pressure was set to be 1 bar, which was maintained by the Monte Carlo Membrane Barostat for membrane systems and by the Monte Carlo Barostat for the $\mathrm{C}_{3}-\mathrm{PC}$ system. For the Monte Carlo Membrane Barostat, a semi-isotropic simulation cell was used, where X was constrained to 
be equal to $\mathrm{Y}$ but allowed to vary independently with respect to $\mathrm{Z}$ (the bilayer normal direction). The real-space cutoff, $r_{\text {cut }}$, was set to be $10 \AA$ in all OpenMM simulations.

After the Linkage optimization, 3 replicas of 100 ns CHARMM (LJ-PME) ${ }^{27}$ NPT simulations were performed for DPPC bilayer at 323.15 K to check the consistency between OpenMM and CHARMM. The Nosé-Hoover thermostat $^{74}$ was used to maintain system temperature, and a modified Andersen-Hoover barostat ${ }^{75,76}$ was $^{7 s e d}$ to maintain constant pressure at 1 bar. The initial structure of the DPPC bilayer was taken from the last frame of the OpenMM simulation at the end of the Linkage optimization. The same semi-isotropic simulation cell and $r_{\text {cut }}$ were used to keep consistency with the OpenMM simulations. The last $70 \mathrm{~ns}$ from each replica was used for the surface area calculation.

Surface area per lipid $\left(A_{l}\right)$ was calculated as the area of the cross-section perpendicular to the membrane normal divided by the number of lipids in each leaflet. The compressibility modulus of bilayer, $K_{\mathrm{A}}$, was determined from the isotherm of the surface tension with respect to the total area ${ }^{25}$

$$
K_{A}=A\left(\frac{d \gamma}{d A}\right)_{T}
$$

where $A$ is the total area, and $\gamma$ is the total surface tension. The deuterium order parameter $\left(S_{\mathrm{CD}}\right)$ was calculated as

$$
S_{\mathrm{CD}}=\left|\frac{1}{2}\left\langle 3 \cos ^{2} \theta-1\right\rangle\right|
$$

where $\theta$ is the angle formed by the $\mathrm{C}-\mathrm{H}$ vector and the bilayer normal, and the average is over the ensemble and time.

To extract the maximum and minimum of $\mathrm{C}_{3}$-PC $\sim$ water RDFs, MDTraj ${ }^{77}$ was used to calculate the 3 dimensional RDFs from simulation. The experimental data was taken from Foglia et al. ${ }^{57}$ Due to the noise in the experimental RDFs, we used the "convolve" function in $\mathrm{NumPy}^{78}$ to smooth the curve with a window size of 3. The RDFs from simulation were generated with enough sampling so that no smoothing was needed.

\section{Results}

The long-range dispersion has a significant impact on membrane structure. The C36 lipid FF was parametrized specifically for a Lennard-Jones functional form of the van der Waals interaction with a force-switching function over the range of 8-12 $\AA,{ }^{14}$ and any deviation from this "standard" scheme is expected to cause different structural 
measurements compared to simulations using this "standard". For example, changing the switching function to 11-12 $\AA$ and simulating in NAMD ${ }^{79}$ instead of CHARMM led to a significantly lower $A_{1}\left(59.1 \pm 0.4 \AA^{2}\right.$ compared to $62.9 \pm 0.3 \AA^{2}$ ) for DPPC bilayer at $323.15 \mathrm{~K} .{ }^{14}$ While the simulation package and relatively short sampling time of the CHARMM simulation (40 ns) might have also contributed to this difference, the $200 \mathrm{~ns}$ simulation at the beginning of the current parametrization also generated a significantly lower $A_{1}\left(58.9 \pm 0.3 \AA^{2}\right)$ when the longrange dispersion was fully included through LJ-PME. Deuterium order parameters were also in poor agreement with experiment as shown in Figure S2. In this section, we show how this degradation in the reproduction of experimental observables and the inconsistency between monolayer and bilayer were fixed.

3.1. Parameter Sensitivities. The sensitivity analysis was performed on all training targets associated with the PC headgroup starting from the first optimization cycle. Sensitivities for the surface area of DPPC bilayer at 323.15 K are reported in Figure 3; examples for other training targets can be found in Figure S3. The sensitivities were calculated according to eq 1 , and the perturbation sizes $\delta \lambda$ were determined by monitoring the effects of the size (see Section 3.2 for more details). A perturbation size of 0.001 elementary charge (e) was chosen for partial charges (about $0.3 \%$ the average absolute value of partial charges from head group atoms), and percentage perturbations $(0.1 \%)$ were used for $R_{\min } / 2$ and $\varepsilon$ due to the wide distributions of these two parameters among different atom types. 
Global
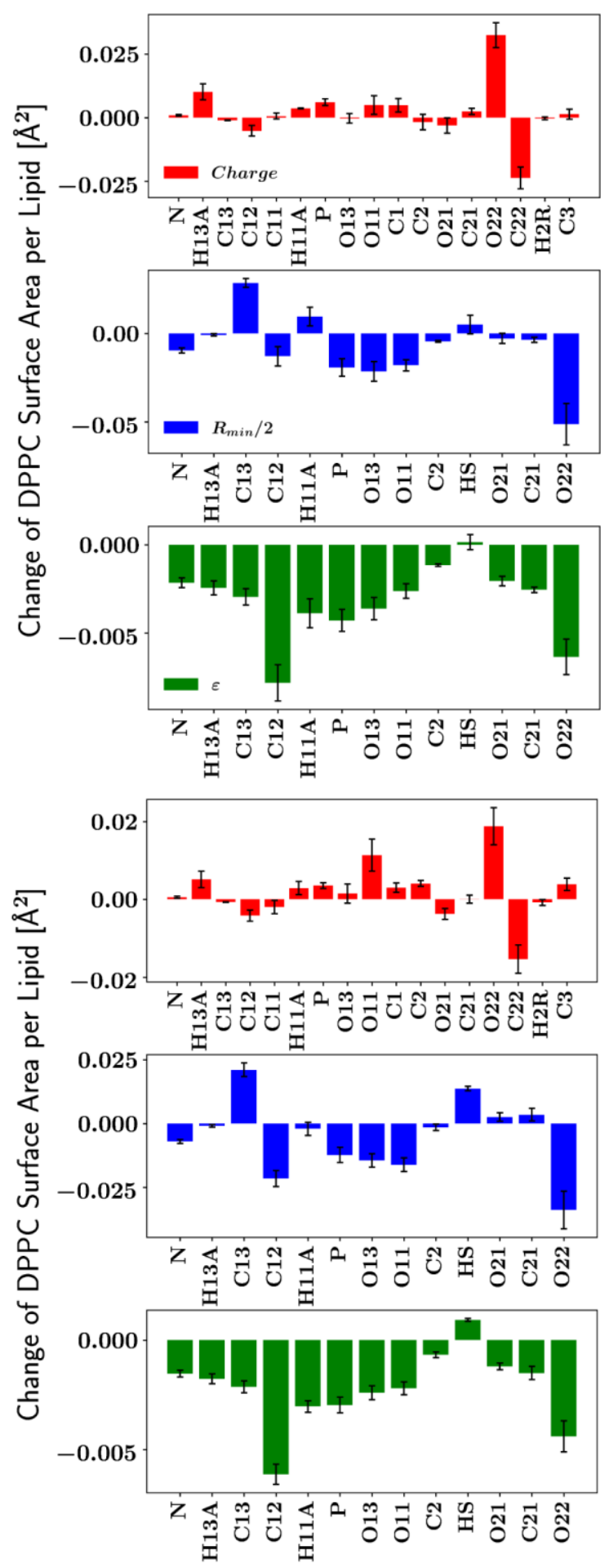
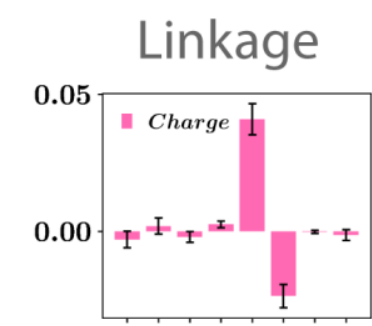

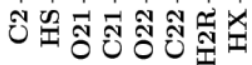
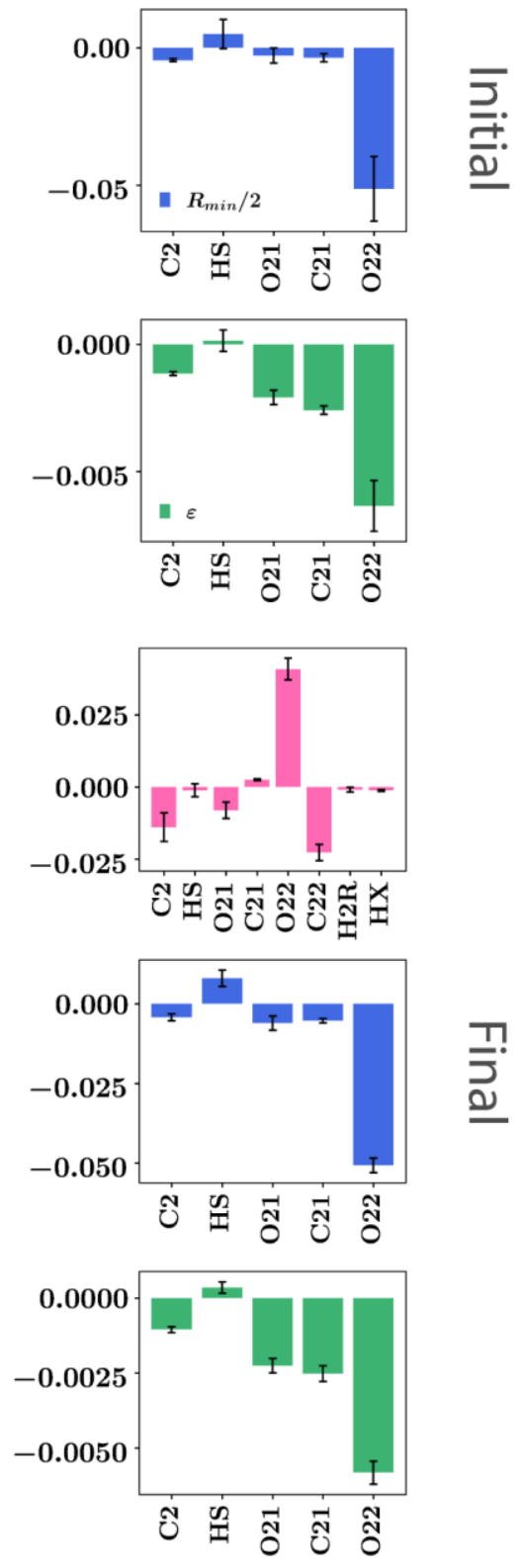

Figure 3. Parameter sensitivities for the surface area of DPPC bilayer at $323.15 \mathrm{~K}$. Averages and standard deviation evaluated from three blocks of $50 \mathrm{~ns}$ shown for each parameter. The perturbation sizes used are 0.001 e (absolute value) for partial charge and $0.1 \%$ of the original parameter for $R_{\min } / 2$ and $\varepsilon$. 
It is evident from Figure 3 that different parameters have different effects on the surface area of DPPC bilayer and its standard deviations. While the perturbations used for the three nonbonded parameter types (charge, $R_{\min } / 2$ and $\varepsilon$ ) are comparable, $\varepsilon$ has much less influence on the surface area compared to the other two, and the sensitivities are generally negative (when increasing the absolute value of $\varepsilon$ ). This is not surprising since $\varepsilon$ does not substantially influence the minimum energy distance between two interacting groups, though the distance distribution for an atom pair is affected by the interplay of all nonbonded parameters (and bonded parameters if linked). The $R_{\min } / 2$ sensitivities do not have a uniform sign, but most are negative. This is counterintuitive as one might expect the volume to increase with larger $R_{\min }$, hence an increased surface area. However, it is the intersection of the LJ surfaces of the two interacting groups that impact the minimum interaction distances such that the relationship of $\mathrm{R}_{\min }$ to interaction distance is not necessarily directly correlated. This result highlights the complexity of interactions in lipid bilayers. For partial charges, the "hot spots" are the two carbonyl groups (C22$\mathrm{O} 22 / \mathrm{C} 32-\mathrm{O} 32$ ) at the head-tail linkage region. Moreover, the $\mathrm{O} 22 R_{\min } / 2$ also has a significant impact on the surface area. Because these atoms are located at the head-tail linkage, two optimizations were performed. As detailed in the Methods, Linkage restricted the changes to the glycerol backbone and the ester groups, while Global also allowed the phosphate and choline groups to vary.

The surface area of DPPC was not the only target for parametrization, and the sensitivity for a single property can only tell us the importance of the parameter for that particular property, but not necessarily others. This is illustrated by Figure S3 and Figure 4. Figure S3 shows additional parameter sensitivities for $A_{l}$ of DMPC bilayer, overall bilayer thickness $\left(D_{\mathrm{B}}\right)$ of a DPPC bilayer, $S_{\mathrm{CD}}$ of carbon C12 in a DPPC bilayer and the first peak of the $\mathrm{RDF}$ between the carbonyl oxygen and the water oxygen of the $\mathrm{C}_{3}-\mathrm{PC}$ solution. The sensitivities for $D_{\mathrm{B}}$ are inversely related to the sensitivities for $A_{l}$, consistent with the compressibility modulus of DPPC bilayers. From the perspective of parametrization, this relationship reveals the essential role hydration plays in the surface area. The sensitivities for $A_{l}$ of DMPC are very similar to those of DPPC, which means the two areas are highly correlated. For the $S_{\mathrm{CD}}$ of carbon $\mathrm{C} 12$, the parameters having substantial influence are not limited to the phosphatecholine link region. Since $S_{\mathrm{CD}}$ is calculated from the $\mathrm{C}-\mathrm{H}$ bond orientation which is primarily determined by the dihedral parameters once the angle parameters were well-determined, it might not be influenced by the nonbonded parameters directly. However, the nonbonded parameters may influence the interactions within or between lipid molecules (and water). Hence, they can change the overall bilayer structure and the orientation of the head group indirectly. 


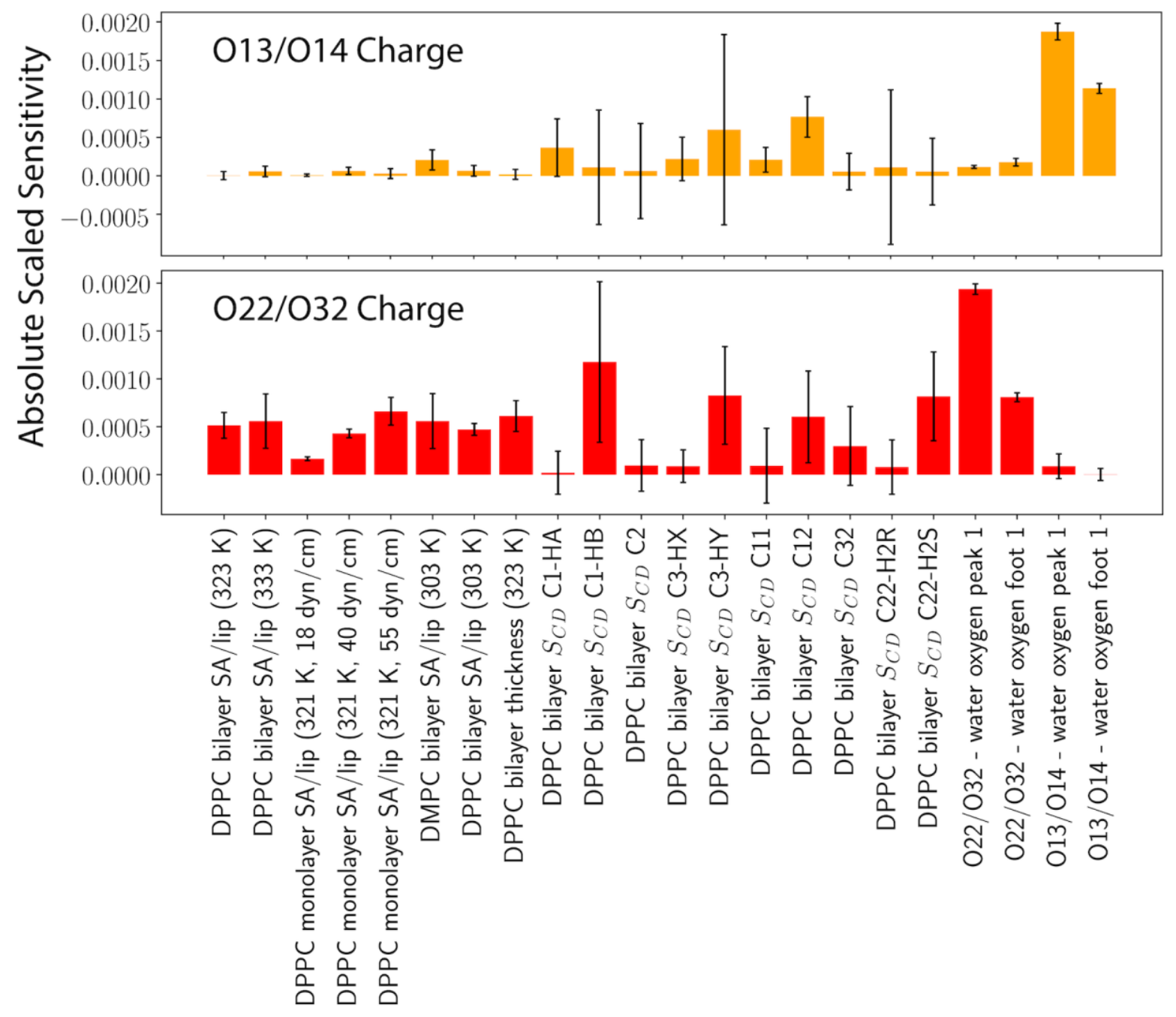

Figure 4. Absolute scaled parameter sensitivities of O13/O14 and O22/O32 partial charges for various properties.

Figure 4 plots the parameter sensitivities of $\mathrm{O} 13 / \mathrm{O} 14$ and $\mathrm{O} 22 / \mathrm{O} 32$ charges on a wide range of properties, which are scaled by the scaling factors in Table 1 after taking the absolute values. The parameter perturbations used are the same as those used in Figure 3. It is clear that the standard deviations of the sensitivities for different properties vary significantly. For example, one can be almost certain about O13/O14 charges' influence on the RDF between O13/O14 and the water oxygen but cannot be so confident with the $A_{l}$ of DPPC/DMPC bilayers. However, when parametrizing the force field, the influence of the $\mathrm{O} 13 / \mathrm{O} 14$ charges on the $A_{l}$ is nonnegligible, which makes the parametrization challenging. 

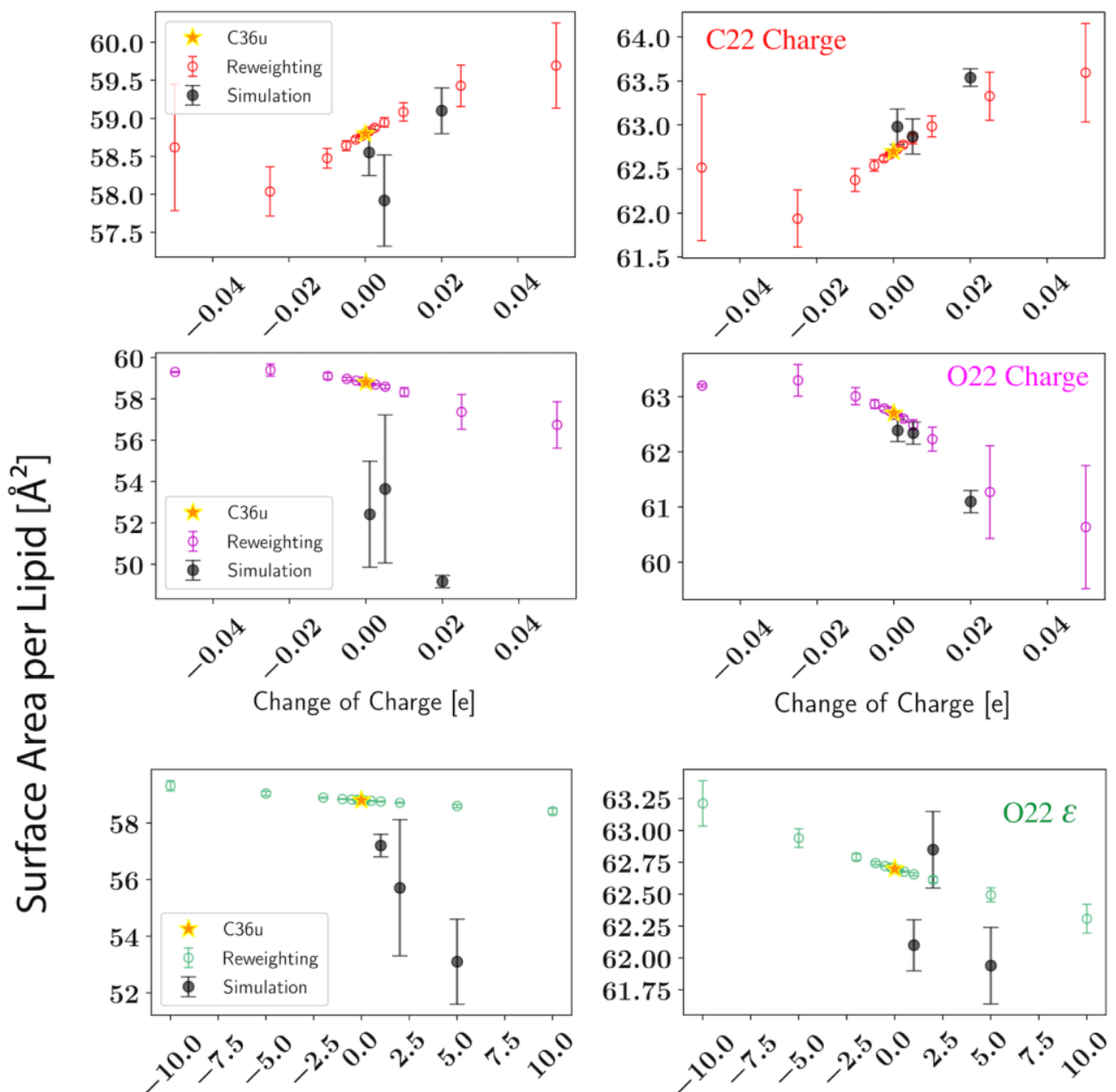

Change of Epsilon [\%]

Change of $\varepsilon[\%]$

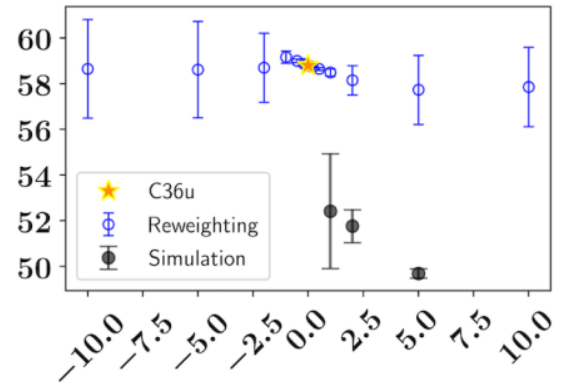

Change of Sigma [\%]

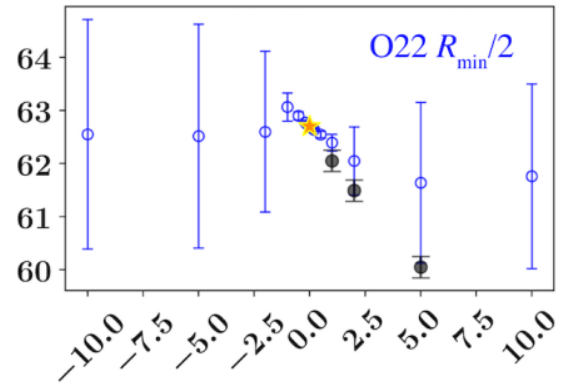

Change of $\boldsymbol{R}_{\min } / 2$ [\%]

Figure 5. Simulated and reweighted surface area per lipid for DPPC bilayer at $323.15 \mathrm{~K}$. All simulations are performed with LJ-PME. Yellow stars $(\mathrm{C} 36 \mathrm{u})$ : simulations using the original C36 parameter set (left) or the final parameter set (right) from the Global optimization; black dots with error bars (Simulation): simulations using perturbed parameters; circles with error bars: predictions by reweighting the yellow-star simulations. The averages and standard deviations shown at error bars of the simulated results are based on 3 independent replicas of $300 \mathrm{~ns}$ (last $200 \mathrm{~ns}$ used for calculating the $A_{l}$ ), while the averages and standard deviations shown at error bars of the reweighted are from 3 trajectory blocks of $50 \mathrm{~ns}$. 
3.2. Scope of the Linear Prediction. As noted in the Method Section, one of the major changes from early parametrizations of the CHARMM lipid FF is the application of thermodynamic reweighting. Although this can potentially increase the efficiency of the parametrization, one should be careful about the parameter sensitivities predicted in this way. The reason is simple - to get meaningful sensitivities (gradients), the trajectory sampled by the simulation should represent an equilibrated state. And perhaps more importantly, the gradients calculated at a certain point in the parameter space are only accurate for a specific region around that point, which is hereafter called the linear region.

To identify the size of the linear region and to justify our reweighting strategy, we performed reweightings using different perturbation sizes; selected results are shown in Figure 5. For both the starting point (C36) and the end point (examples only shown for the Global parametrization), there are clearly linear regions, but the size of the linear region depends on the parameter. In the view of reweighting, the linear region is typically several hundredths of the elementary charge for partial atomic charges. Interestingly, this region is about $3 \%$ for $R_{\min } / 2$ but more than $10 \%$ for $\varepsilon$. Moreover, the statistical errors calculated based on 3 trajectory blocks indicate that reweighting for $R_{\min } / 2$ is no longer accurate when the perturbation exceeds $3 \%$. Considering $R_{\min } / 2$ is directly related to the pairwise interaction between two atoms close to each other, changing $R_{\min } / 2$ would lead to dramatic change in the conformational sampling so that the overlap between the sampled and reweighted states will be greatly reduced, thus leading to large statistical errors. As a result, we set the weight factors for regularization differently for the three parameter types. See Section 3.3 for more details.

The linear region is harder to detect from direct simulations due to the large statistical errors, while the physics-informed method, reweighting, can greatly reduce the noise in the first derivative. Nevertheless, complications can arise. For example, several simulations near the C36 parameter set condensed to the gel phase leading to huge statistical errors on the mean surface area. Since this only happened to the perturbed set, the microstates representing the gel phase were not captured by the simulation with the C36 set (the one used to reweight). Hence, the formation of a gel phase was not predicted by reweighting. Ideally, one would like to avoid such a scenario where the trajectory used to reweight does not sample some of important regions of the target state. However, the energy landscape of a lipid bilayer is so complex that it is almost certain to be nonergodic. Fortunately, as the optimization went on, the gel phase region of the parameter space was no longer sampled. Therefore, we can have more confidence in the reweighting. This is evident from the bottom row of Figure 5, where the reweightings agree well with the simulations, at least within the linear region.

3.3. Perturbation Sizes and Optimization Weights. Based on the linear region detected in Section 3.2, the perturbation sizes for the three parameter types can be readily determined. To ensure a well-behaved computation of sensitivities, perturbations were set to be much smaller than the sizes of the linear region (see Table 3). As 
noted in the Section 2.5, regularization on the parameter change is necessary to avoid overshooting and can be achieved by including the parameter change into the objective function through a user-defined weight factor and a sensitivity of 1. Based on the results of Section 3.2 and a set of tests on different values, the default weight factors were determined to be 4/e for charges, and 6/percentage-change for $\varepsilon$ and $R_{\min } / 2$ in the first optimization cycle, because they generated parameter changes within the linear region. Apart from the default values, we computed a candidate weight factor $w_{\lambda}$ for parameter $\lambda$ according to eq 6 and used it instead when larger than the default. Lastly, when $w_{\lambda}$ was larger than 20/e for charge or 20/percentage for $\varepsilon$ and $R_{\min } / 2$, the weight factor for parameter $\lambda$ was set to be infinite, since a high $w_{\lambda}$ indicates large noise in the reweighting.

3.4. Dihedral Parameter Fitting. The dihedrals were fitted to either QM or C36. Conformational energies were obtained for $\alpha 1, \alpha 4$ and $\gamma 3$ from C36 by scanning the dihedrals of interest from $-180^{\circ}$ to $180^{\circ}$ in increments of $2^{\circ}$, while other dihedrals were relaxed during the scan to get the minimum energy conformation. QM energies from ref ${ }^{14}$ were used for $\beta 4$ and $\gamma 4$. The scan of $\beta 4$ was from $-180^{\circ}$ to $0^{\circ}$ and the scan of $\gamma 4$ was from $0^{\circ}$ to $180^{\circ}$. Since the two dihedrals contain the same four atom types, the fitting was then conducted by giving each dihedral an equal weight. For dihedrals closer to the glycerol region, reweightings were used to match the C36 dihedral distributions based on DPPC bilayer simulations at $323.15 \mathrm{~K}$. In addition to the standard dihedral fittings for each optimization cycle shown in Table 2, additional adjustments were made to N-C12-C12-O12 ( $\left.\alpha_{5}\right)$ and $\alpha_{4}$ at the end of the Linkage optimization to match the experimental order parameters of carbon $\mathrm{C} 11$ and $\mathrm{C} 12$. The two torsions were selected by checking the correlations between the order parameters and all the head group torsions. Then, the same procedure used for the nonbonded parametrization was used to optimize the torsions (reweighting + least-square fitting) because only small changes were expected. Multiplicities of all dihedrals were kept as they were in C36 for Linkage but allowed to expand for Global. The PES of dihedrals optimized through direct fits are plotted in Figure 6(a), in which the increments for $\alpha 1, \alpha 4$ and $\gamma 3$ are $10^{\circ}$ while the increments for $\beta 4$ and $\gamma 4$ are those from the original scan. Figure 6(b) illustrates the quality of the reweighting for the last optimization cycle. Overall, the optimized set agrees very well with C36. 

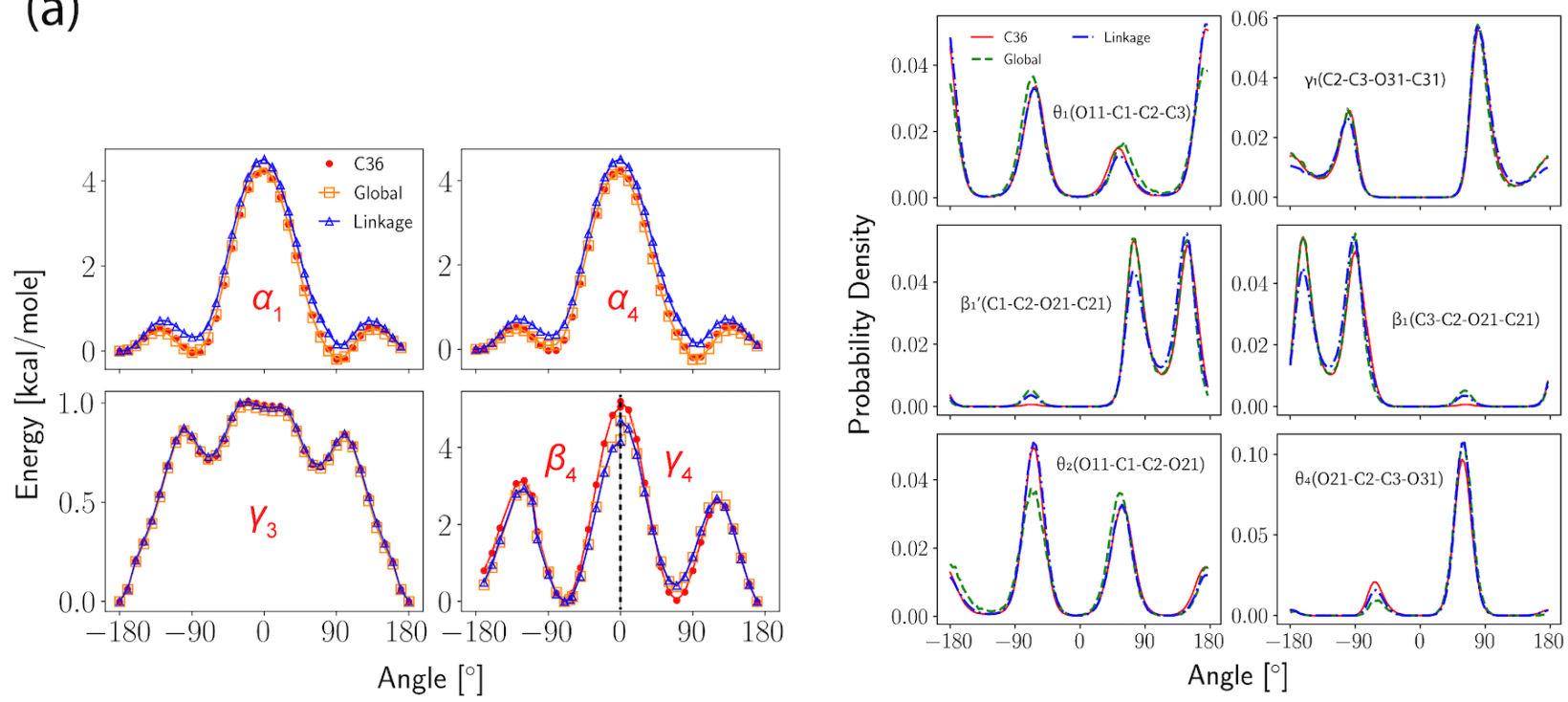

Figure 6. Dihedral fits. (a) Potential energy scans for $\alpha 1, \alpha 4, \gamma 3, \beta 4$ and $\gamma 4$. Reference energy is set to be the lowest energy of the Linkage parametrization; (b) Dihedral angle distributions for $\theta_{1}, \gamma_{1}, \beta_{1}{ }^{\prime}$ (and $\beta_{1}$ ), $\theta_{2}$ and $\theta_{4}$. The simulation used to reweight is not shown.

3.5. Training Set Evolution. Table 4 presents the evolution of the level of agreement with the training set during the two optimizations. At the beginning, $A_{l}$ 's for bilayers were systematically underestimated while the membrane thicknesses were overestimated. Hence, the attractive force brought by the long-range LJ interactions must have a stronger effect in the $x$-y plane (the membrane normal is $z$ ), which can be explained by the heterogeneity of the membrane system. Underestimation of the monolayer surface area was more severe, which was expected since monolayers are not well modeled by the C36 parameter set. The first optimization cycle significantly improved the $A_{l}$ for both bilayer and monolayer, while the remaining cycle(s) brought the DPPC bilayer properties $\left(A_{l}\right.$, membrane thickness, and $\left.S_{\mathrm{CD}}\right)$ closer to the experiments, because the weight factors for DPPC bilayer related properties were overall higher than the other properties. In addition to the OpenMM results reported in Table 4, CHARMM simulations of DPPC bilayer at 323.15 generated an $A_{l}$ of $62.8 \pm 0.2 \AA^{2}$, which is statistically identical to the OpenMM result. Table 4 also shows that the overall quality of Linkage is comparable to Global, and differences between the two are generally within the statistical errors. To minimize the change to the C36 FF and to maximize the consistency of the FF, the Linkage parametrization is chosen as the LJ-PME version of the CHARMM lipid FF and will be called C36/LJ-PME henceforth. 
Table 4. Training set (percentage) deviations from experimental values. "G-1" can be interpreted as "after the first cycle of the Global parametrization" and "L" stands for the Linkage parametrization. Simulated values using the final parameter sets are also reported with standard errors.

\begin{tabular}{|l|l|l|l|l|l|l|l|}
\hline property & expt. & G/L-0 & G-1 & G-2 & G-3 & L-1 & L-2 (C36/LJ-PME) \\
\hline$A_{l}$, DPPC bilayer $(323.15 \mathrm{~K})$ & $63.1 \AA^{2}$ & $-6.7 \%$ & $-2.1 \%$ & $1.4 \%$ & $-0.5 \%\left(62.8 \pm 0.2 \AA^{2}\right)$ & $-2.9 \%$ & $-0.63 \%\left(62.7 \pm 0.2 \AA^{2}\right)$ \\
\hline$A_{l}$, DPPC bilayer $(333.15 \mathrm{~K})$ & $65.0 \AA^{2}$ & $-7.3 \%$ & $-2.3 \%$ & $-0.1 \%$ & $-1.8 \%\left(63.8 \pm 0.2 \AA^{2}\right)$ & $-4.2 \%$ & $-2.6 \%\left(63.3 \pm 0.2 \AA^{2}\right)$ \\
\hline$A_{l}$, DMPC bilayer $(303.15 \mathrm{~K})$ & $60.6 \AA^{2}$ & $-6.4 \%$ & $2.0 \%$ & $4.2 \%$ & $3.3 \%\left(62.6 \pm 0.4 \AA^{2}\right)$ & $-0.8 \%$ & $1.8 \%\left(61.7 \pm 0.2 \AA^{2}\right)$ \\
\hline$A_{l}$, POPC bilayer $(303.15 \mathrm{~K})$ & $64.4 \AA^{2}$ & $-3.9 \%$ & $2.3 \%$ & $4.1 \%$ & $2.2 \%\left(65.8 \pm 0.4 \AA^{2}\right)$ & $0.4 \%$ & $1.9 \%\left(65.6 \pm 0.4 \AA^{2}\right)$ \\
\hline chain $S_{C D}$ of DPPC bilayer (averaged) & N/A & $26.5 \%$ & $12.1 \%$ & $6.8 \%$ & $8.4 \%$ & $14.9 \%$ & $13.7 \%$ \\
\hline$D_{B}$, DPPC bilayer $(323.15 \mathrm{~K})$ & $39.0 \AA$ & $5.6 \%$ & $1.9 \%$ & $-1.4 \%$ & $-0.3 \%(38.9 \pm 0.3 \AA)$ & $1.8 \%$ & $-1.3 \%(38.5 \pm 0.1 \AA)$ \\
\hline$D_{B}$, DPPC bilayer $(333.15 \mathrm{~K})$ & $38.1 \AA$ & $6.2 \%$ & $1.6 \%$ & $0.2 \%$ & $0.3 \%(38.2 \pm 0.1 \AA)$ & $2.8 \%$ & $0.5 \%(38.3 \pm 0.1 \AA)$ \\
\hline$D_{B}$, DMPC bilayer $(303.15 \mathrm{~K})$ & $36.7 \AA$ & $3.4 \%$ & $-4.4 \%$ & $-6.7 \%$ & $-5.2 \%(34.9 \pm 0.4 \AA)$ & $-2.6 \%$ & $-5.7 \%(34.6 \pm 0.2 \AA)$ \\
\hline$A_{l}$, DPPC monolayer $(18 \mathrm{dyn} / \mathrm{cm})$ & $54.0 \AA^{2}$ & $-9.0 \%$ & $1.9 \%$ & $4.9 \%$ & $4.2 \%\left(56.3 \pm 0.2 \AA^{2}\right)$ & $1.4 \%$ & $3.1 \%\left(55.7 \pm 0.2 \AA^{2}\right)$ \\
\hline$A_{l}$, DPPC monolayer $(40 \mathrm{dyn} / \mathrm{cm})$ & $64.0 \AA^{2}$ & $-5.5 \%$ & $-0.5 \%$ & $2.3 \%$ & $0.8 \%\left(64.5 \pm 0.3 \AA^{2}\right)$ & $-1.5 \%$ & $0.1 \%\left(63.9 \pm 0.2 \AA^{2}\right)$ \\
\hline$A_{l}$, DPPC monolayer $(55 \mathrm{dyn} / \mathrm{cm})$ & $80.0 \AA^{2}$ & $-11.4 \%$ & $-5.4 \%$ & $-1.9 \%$ & $-4.1 \%\left(76.7 \pm 0.3 \AA^{2}\right)$ & $-7.2 \%$ & $-4.4 \%\left(76.5 \pm 0.3 \AA^{2}\right)$ \\
\hline$K_{A}$, DPPC bilayer at $323.15 \mathrm{~K}$ & $0.23 \mathrm{~N} / \mathrm{m}$ & $4 \%$ & $34.8 \%$ & $17.4 \%$ & $0 \%(0.23 \pm 0.04 \mathrm{~N} / \mathrm{m})$ & $30.4 \%$ & $4.3 \%(0.24 \pm 0.02 \mathrm{~N} / \mathrm{m})$ \\
\hline
\end{tabular}

The $S_{\mathrm{CD}}$ profiles of DPPC bilayer are shown in Figure 7(a). The optimized FFs are in excellent agreement with experiment for both head group and tails. While the chain $S_{\mathrm{CD}}$ is strongly correlated with $A_{l}{ }^{80}$ the accurate $S_{\mathrm{CD}}$ of the head group indicates that the reweighting for the dihedral parameters, especially those from the glycerol region, was a success. In addition to the unsigned deuterium order parameter $\left(S_{\mathrm{CD}}\right)$, the ${ }^{13} \mathrm{C}-{ }^{1} \mathrm{H}$ dipolar order parameter $\left(S_{\mathrm{CH}}\right)$ can be measured experimentally, ${ }^{81}$ which comes with a sign

$$
S_{\mathrm{CH}}=\frac{1}{2}\left\langle 3 \cos ^{2} \theta-1\right\rangle
$$

where $\theta$ is the angle formed by the $\mathrm{C}-\mathrm{H}$ vector and the bilayer normal. Although experiments measuring the sign of this quantity are limited, ${ }^{81}$ they can provide insightful information regarding the structure of the head group and resolve ambiguity in the sign of the deuterium order parameter. The comparison between simulation and experiment is shown in Figure 7(b). In fact, the optimized FFs correctly obtain the sign for all the head group carbons even though these signs were not considered in the original parametrization of C36. In essence, the highlevel QM conformational energies chosen as the starting points for dihedral parametrization of C36 led to the good agreement with experiment. 


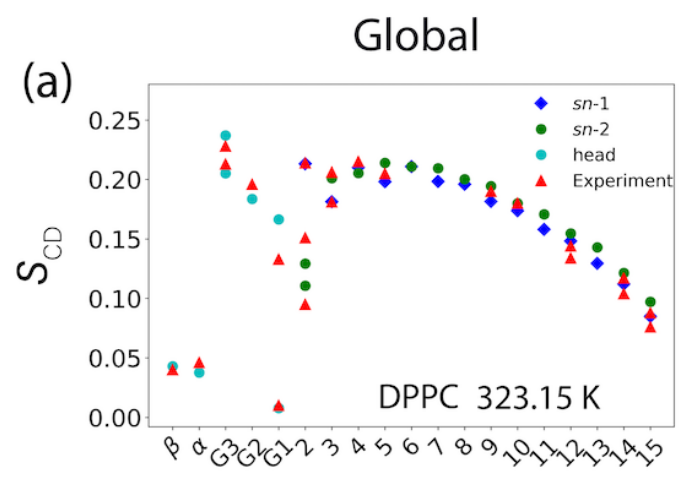

Linkage (C36/LJ-PME)

(b) $\quad \begin{aligned} & \text { C36/L-PME (DMPC) } \\ & \text { Expt.1 (DMPC) Expt. } 2 \text { (egg lecithin) }\end{aligned}$

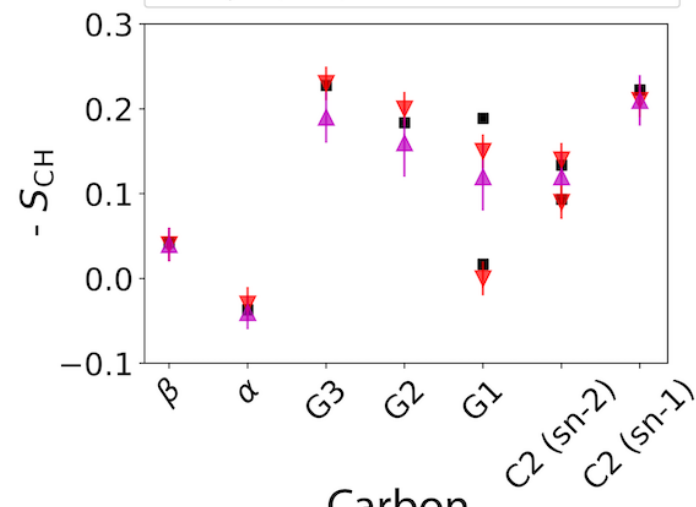

Carbon

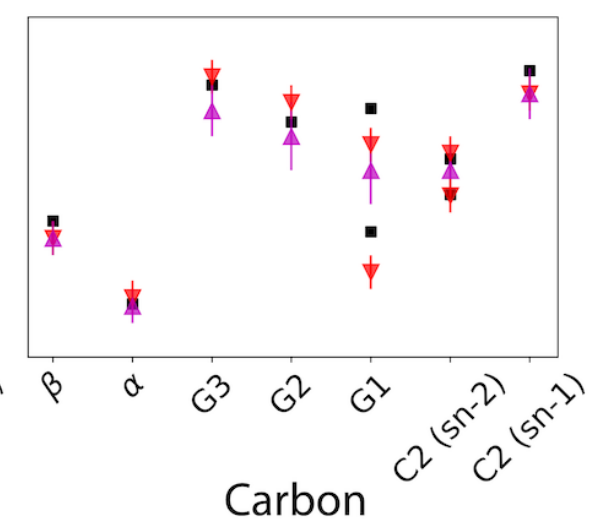

Figure 7. (a) Deuterium order parameters $\left(S_{\mathrm{CD}}\right)$ of DPPC bilayer at $323.15 \mathrm{~K}$. (b) ${ }^{13} \mathrm{C}^{-1} \mathrm{H}$ dipolar order parameters $\left(S_{\mathrm{CH}}\right)$ from simulations of DMPC bilayer and two experiments (DMPC ${ }^{81}$ and egg yolk lecithin ${ }^{82}$ ).

The RDFs between water and $\mathrm{C}_{3}-\mathrm{PC}$ molecules are in principle important metrics for binding strength and structure of water molecules around the lipid head. However, the water model (TIP3P) used, the additive formalism of the potential energy, and the uncertainty in the interpretation of the experimental data led us to assign relatively low weights to the RDF. Figure 8 plots the RDFs for the two optimizations, as well as C36. Global improved the RDFs for the phosphate ester oxygens (O11/O12), as their first peaks decreased toward the experiment. This trend was not observed for Linkage (C36/LJ-PME), because nonbonded parameters of the phosphate group were not allowed to change. The ester groups located at the head-tail link region were barely influenced in both optimizations. This is probably a consequence of the competition between the RDF and the surface area (more hydration in the head group region was needed to expand the membrane in the $x-y$ plane). However, there was a shift to the left for the O22/O32 peaks, which was directly due to the decreased van der Waals radius. 

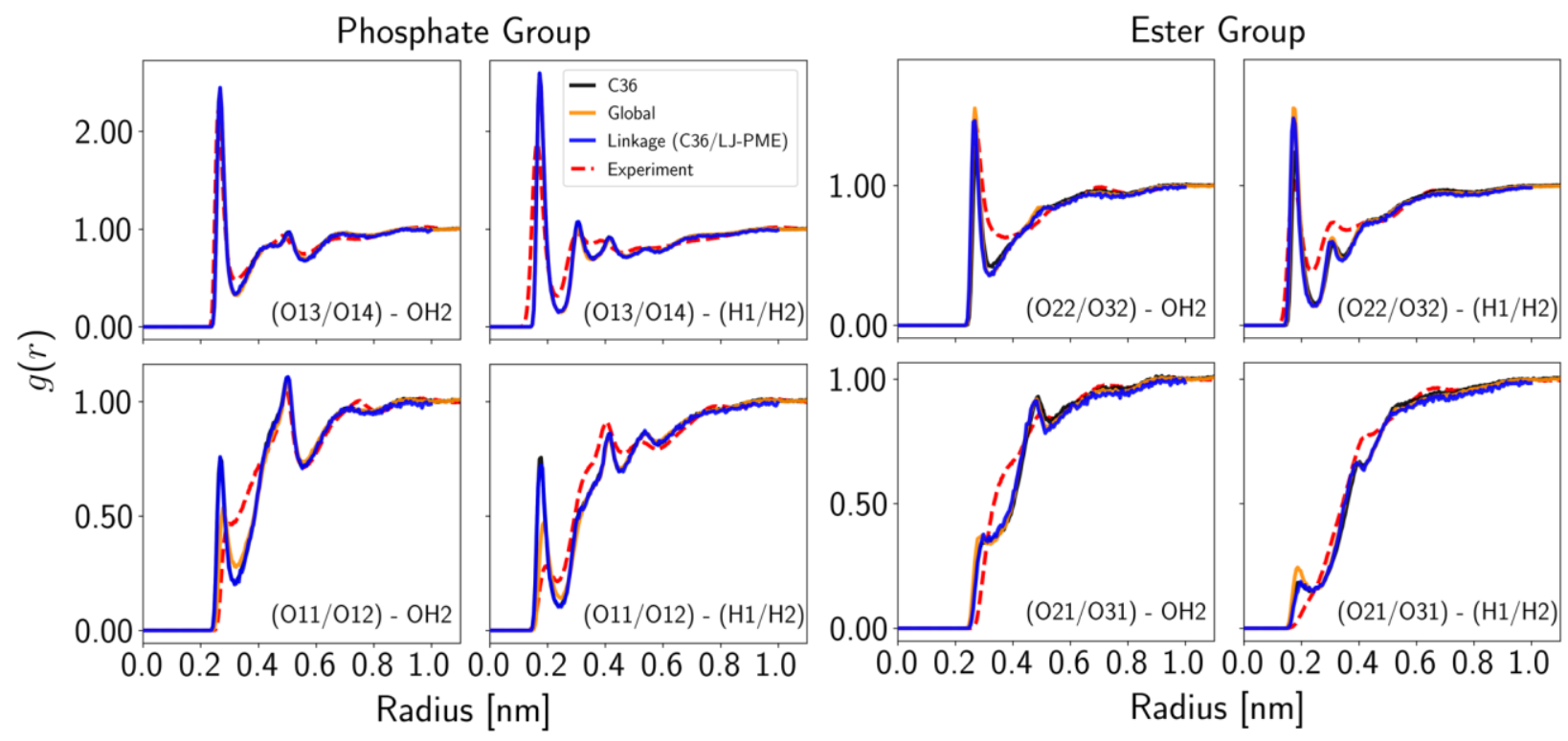

Figure 8. Atom-pair-specific RDFs between water and $\mathrm{C}_{3}-\mathrm{PC}$ in solution for each optimization cycle.

3.6 Parameter Evolution. For both optimizations, only nonbonded parameters for the PC head were adjusted. Evolution of these nonbonded parameters are presented in Table S5-S7. In Global, changes in the last two optimization cycles are in the same direction as the first cycle but with smaller sizes for most partial atomic charges. For $\varepsilon$, changes are only seen in the first and the second optimization cycles and mostly in the direction of decreasing the well depth. However, no change is observed for the last optimization cycle because of the larger weightings and the relatively weak sensitivities. The most interesting part is the oscillation of $R_{\min } / 2$ for $\mathrm{O} 21 / \mathrm{O} 31$ and $\mathrm{O} 22 / \mathrm{O} 32$, which are the ester and carbonyl oxygens from the ester linkage. Since the O22/O32 $R_{\min } / 2$ has a great influence on the surface area, the oscillation is consistent with the change in the surface area (Table 4). However, the oscillation in $\mathrm{O} 21 / \mathrm{O} 31 \mathrm{R}_{\mathrm{min}} / 2$ is probably more stochastic because of its weak sensitivity. $R_{\min } / 2$ for O13/O14 was modified after cycle 2 according to our interim validation on PG lipids (not published) so that it should be viewed differently. In Linkage, there are more oscillations in the partial charges between the two cycles rather than the consistent trends observed in Global, but the relatively small changes in the second optimization cycle indicate that the final parameter set is very close to the local optimum. In terms of LJ parameters, the biggest changes are for the $\mathrm{O} 22 / \mathrm{O} 32 R_{\mathrm{min}} / 2$ (decrease) and the $\mathrm{O} 21 / \mathrm{O} 31 R_{\min } / 2$ (increase), which improve the surface area and the RMSD of RDF, respectively. To compare the partial charges from the two optimizations, Figure 9 plots their changes with respect to C36. Overall, the changes are small. The most evident differences are for HS, C3, O22/O32. While the first two can be explained by the different rules of charge transfer during the optimization (Table S2) and their relatively weak influences on the surface area, the difference in $\mathrm{O} 22 / \mathrm{O} 32$ is more likely a consequence of constraining the parametrization to the linkage region and the use of RDF RMSD as the fitting target, which led to more decrease in the O22/O32 $R_{\min } / 2$ in Linkage. Since the decrease 
in the $\mathrm{O} 22 / \mathrm{O} 32 R_{\mathrm{min}} / 2$ has a similar effect as the decrease in the $\mathrm{O} 22 / \mathrm{O} 32$ charge (in terms of the surface area), less change in the $\mathrm{O} 22 / \mathrm{O} 32$ charge is needed in the end.

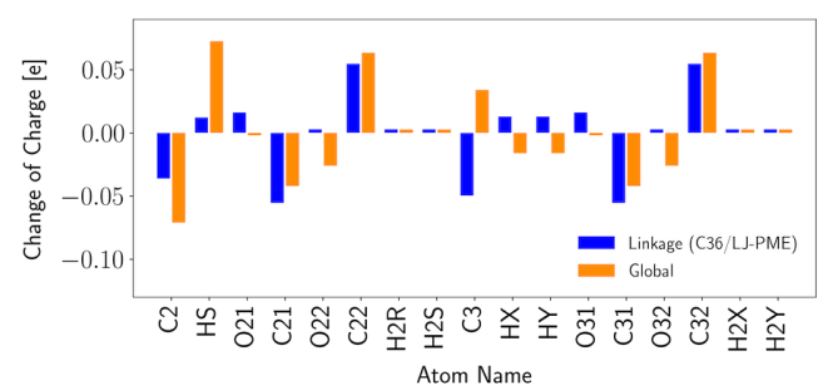

Figure 9. Partial charge changes relative to C36. Only parameters from the linkage region are presented.

In principle, the optimization protocol can be set to terminate automatically according to a preset convergence criterion, for example, a threshold for a function using the relative error of the target properties and the change of the parameters as inputs. However, there are many properties involved in this parametrization and, therefore, determining a general meaningful threshold beforehand is not practical. This led us to use human interruptions when the properties are satisfactory according to our experience. However, the program can be easily modified to allow automatic termination if needed.

\section{Discussion and Conclusions}

In this paper, a semi-automated approach for optimizing the CHARMM additive lipid FF is presented. The motivation for the optimization is to incorporate long-range Lennard-Jones terms into the FF to obtain consistent surface tensions for lipid bilayers and monolayers, thereby resolving the inconsistency in C36 in the treatment of mono- versus bilayers. The optimization focused on the nonbonded parameters of the PC head group and associated torsions. Unlike the early developments of the CHARMM lipid FF, which started from parametrizations of small model compounds, our method additionally takes advantage of thermodynamic reweighting to estimate the first derivatives of membrane-related properties with respect to parameters of interest and iteratively changes the parameters to generate better predictions for these properties. Compared to the conventional method, which may take months or longer to parametrize a single lipid, the new method only takes a few weeks to find high-quality parameters for several lipids at the same time, and it can be modified easily to include more lipid types as long as experimental data are available.

Due to the large number of parameters, FF parametrizations are often underdetermined. In the current study, we avoid this by using parameter restraints. However, the choice of weight factors and the statistical errors from both simulation and experiment can still introduce arbitrariness in a fully automated optimization. Therefore, we 
adopt a semi-automated approach in which we can monitor the optimization in real time and adjust the weight factors and parameter restraints if needed. Such manual interruptions do not influence the speed of optimization if the workflow is set up properly.

Despite the efficiency of the method, it should be used with care if there is a potential phase change, a concern in lipid bilayer simulations, or a large structural change when parametrizing a macromolecular FF. In such cases, the trajectory sampled by the simulation may not contain enough or any useful information of the target state, so that a meaningful evaluation of the parameter sensitivity is not guaranteed. It should also be noted that most gradient-based optimization algorithms are only capable of finding the local minimum rather than the global minimum. In other words, the user must have an informed initial guess for the parameters to initiate the optimization. However, once having one, the method can be applied to improve the force field in agreement with newly published experimental data or state-of-the-art simulation protocols such as LJ-PME. Here the C36 parameter set was an excellent starting point because it was parametrized carefully to match experimental measurements of various membrane properties and adding LJ-PME to it will does not represent a large change in the energy function, though it does require the parameters to be adjusted to maintain agreement with experimental data. Although not tested, another a potentially good starting point could be a set of user-defined parameters that match high-level QM calculations for the building blocks of lipids with additional parametrization to include intramolecular parameters combining these blocks (a commonly accepted procedure of modern FF development).

When using the method, settings in the reweighting and the optimizer have to be carefully considered. First, because of the non-linear nature of the optimization problem, perturbation size used in the reweighting should be carefully determined by checking the behavior of the property with respect to the parameter changes in order to generate meaningful first derivatives. This can be done at both the reweighting and the simulation level. While additional simulations can provide direct measurements to avoid insufficient conformational overlapping which can cause trouble in the reweighting, they usually come with larger statistical errors so that using them can be a waste of computational resources. When using direct reweighting, the uncertainty of sensitivity can be estimated using trajectory blocks. After obtaining the sensitivity, the optimizer should be set properly to prevent unreasonably large changes of the parameters. This can be achieved by adding restraints to the parameters. The restraints in our protocol are determined by checking the size of the linear region and the standard deviation of the parameter sensitivity, and by enforcing consistency with the CHARMM FF as a whole.

A linear matrix equation is used to predict the iterative refinement of the nonbonded parameter set in this study. For dihedral optimizations, the BFGS algorithm is used, which searches the parameters by estimating the Hessian matrix based on the first derivatives. Considering the fact that the number of nonbonded parameters is larger than the number of observables, the linear equation is underdetermined without extra restraints. However, by adding parameter restraints, it becomes overdetermined so that a least-squares solution can be found. An 
alternative method to this can be the Levenberg-Marquardt algorithm, ${ }^{44,52}$ which applies a damping term to the parameter change when doing a least-squares fit. In our case, the parameters are not expected to change dramatically so that a linear approximation to the first derivative can be used. However, when searching in a larger parameter space or in certain situations where it is hard to get meaningful parameter sensitivities, derivative-free algorithms such as the simplex method should be considered. ${ }^{53}$

After two or three optimization cycles, parameters were obtained that allow for the CHARMM lipid FF to be used in conjunction with LJ-PME. This represents an important breakthrough as it eliminates the sensitivity of the FF to the specific treatment of truncation of the LJ terms, including how to treat the long-range dispersion contributions. The resulting parameter set yielded strong agreement with a number of experimental observables. $S_{\mathrm{CD}}$ and $S_{\mathrm{CH}}$ are in good agreement with experiment for both the head group and the tail regions. The optimization also takes the atom-pair-specific RDFs between $\mathrm{C}_{3}-\mathrm{PC}$ and water into consideration and improvements are observed. Most importantly, C36/LJ-PME achieves very good agreement with experimental DPPC monolayer pressure/area isotherm, while the accuracy for bilayer surface area is maintained with respect to the original C36 force field without LJ-PME. This is a significant improvement over C36. Monolayer simulations with C36/LJPME can now be compared directly with experiment without adjustment for long-range Lennard-Jones interactions, thereby greatly expanding the kinds of lipid systems that can be examined. Specifically, monolayers are often stable at lipid concentrations, temperatures, $\mathrm{pH}$ and ionic strengths that bilayers are not. For example, the molar fraction of the highly charged lipid, phosphatidylinositol 4,5-bisphosphate $\left(\mathrm{PIP}_{2}\right)$, is typically less than $5 \%$ on average in cell membrane, ${ }^{83}$ though concentrations in local clusters can be much higher. ${ }^{84}$ In experiment, the limit for PIP2 incorporation into bilayer vesicles with PC lipids is approximately $20 \% .{ }^{85,86}$ Cholesterol has a stabilizing effect on PIP2 vesicles, but this requires a minimum cholesterol fraction of $20 \mathrm{~mol} \% .{ }^{86}$ In contrast, monolayers can be formed with $100 \% \mathrm{PIP}_{2} \cdot{ }^{87-89}$ Another example is phosphatidylethanolamine (PE), for which pure monolayers can be formed ${ }^{90,91}$ but bilayers only exist in a narrow range of hydration. ${ }^{92}$ In fact, the large spontaneous curvature of PE promotes the inverse hexagonal $\left(\mathrm{H}_{\mathrm{II}}\right)$ phase under more physiologically relevant conditions (in this case, full hydration)..$^{92}$

The revised force field will be referred to as C36/LJ-PME. To minimize the change with respect to the C36 lipid FF and to maximize the consistency of the CHARMM FF, the Linkage parametrization will be the formal LJ-PME version of the C36 lipid FF and will be available through the program CHARMM, Klauda web site and http://mackerell.umaryland.edu/charmm_ff.shtml. The result from the Global parametrization can be accessed by contacting the authors. While this paper focuses on the optimization problem, Paper II will provide a more extensive validation for the new parameter set. 


\section{Supporting Information Available:}

Table detailing nonbonded parameters changed in the two optimizations; table describing the rules for charge redistribution; table listing the default weight factors for different parameter types; table listing the scaling factor $S_{\mathrm{b}}$ in each optimization cycle; tables listing initial and final nonbonded parameters in both optimizations; figure containing atom notation of the PC head group; figure of $S_{\mathrm{CD}}$ for DPPC bilayer (unoptimized); and figure showing examples of parameter sensitivity.

\section{Acknowledgements:}

The authors thank Jing Huang, Frank C. Pickard IV and Benoît Roux for helpful discussions. This research was supported by the Intramural Research Program of the NIH, the National Heart, Lung, and Blood Institute, and the use of the high-performance computational capabilities at the National Institutes of Health, Bethesda, MD (NHLBI LoBoS and Biowulf clusters). JBK acknowledges support from NSF (CHE-2003912) and ADM acknowledges support from the NIH (GM131710).

\section{References:}

1. Antila, H.; Buslaev, P.; Favela-Rosales, F.; Ferreira, T. M.; Gushchin, I.; Javanainen, M.; Kav, B.; Madsen, J. J.; Melcr, J.; Miettinen, M. S.; Määttä, J.; Nencini, R.; Ollila, O. H. S.; Piggot, T. J., Headgroup Structure and Cation Binding in Phosphatidylserine Lipid Bilayers. The Journal of Physical Chemistry B 2019, 123 (43), 9066-9079.

2. Enkavi, G.; Javanainen, M.; Kulig, W.; Róg, T.; Vattulainen, I., Multiscale Simulations of Biological Membranes: The Challenge To Understand Biological Phenomena in a Living Substance. Chemical Reviews 2019, 119 (9), 5607-5774.

3. Marrink, S. J.; Corradi, V.; Souza, P. C. T.; Ingólfsson, H. I.; Tieleman, D. P.; Sansom, M. S. P., Computational Modeling of Realistic Cell Membranes. Chemical Reviews 2019, 119 (9), 6184-6226.

4. Venable, R. M.; Krämer, A.; Pastor, R. W., Molecular Dynamics Simulations of Membrane Permeability. Chemical Reviews 2019, 119 (9), 5954-5997. 
5. Pluhackova, K.; Kirsch, S. A.; Han, J.; Sun, L.; Jiang, Z.; Unruh, T.; Böckmann, R. A., A Critical Comparison of Biomembrane Force Fields: Structure and Dynamics of Model DMPC, POPC, and POPE Bilayers. The Journal of Physical Chemistry B 2016, 120 (16), 3888-3903.

6. Monje-Galvan, V.; Klauda, J. B., Modeling Yeast Organelle Membranes and How Lipid Diversity Influences Bilayer Properties. Biochemistry 2015, 54 (45), 6852-6861.

7. Yu, Y.; Klauda, J. B., Modeling Pseudomonas aeruginosa inner plasma membrane in planktonic and biofilm modes. The Journal of Chemical Physics 2018, 149 (21), 215102.

8. Muller, M. P.; Jiang, T.; Sun, C.; Lihan, M.; Pant, S.; Mahinthichaichan, P.; Trifan, A.; Tajkhorshid, E., Characterization of Lipid-Protein Interactions and Lipid-Mediated Modulation of Membrane Protein Function through Molecular Simulation. Chemical Reviews 2019, 119 (9), 6086-6161.

9. Stansfeld, P. J., Computational studies of membrane proteins: from sequence to structure to simulation. Current Opinion in Structural Biology 2017, 45, 133-141.

10. Jensen, M. Ø.; Jogini, V.; Borhani, D. W.; Leffler, A. E.; Dror, R. O.; Shaw, D. E., Mechanism of Voltage Gating in Potassium Channels. Science 2012, 336 (6078), 229.

11. Li, Q.; Wanderling, S.; Paduch, M.; Medovoy, D.; Singharoy, A.; McGreevy, R.; Villalba-Galea, C. A.; Hulse, R. E.; Roux, B.; Schulten, K.; Kossiakoff, A.; Perozo, E., Structural mechanism of voltagedependent gating in an isolated voltage-sensing domain. Nature Structural \& Molecular Biology 2014, 21 (3), 244-252.

12. Miao, Y.; Nichols, S. E.; Gasper, P. M.; Metzger, V. T.; McCammon, J. A., Activation and dynamic network of the M2 muscarinic receptor. Proceedings of the National Academy of Sciences 2013, 110 (27), 10982.

13. Jämbeck, J. P. M.; Lyubartsev, A. P., Derivation and Systematic Validation of a Refined All-Atom Force Field for Phosphatidylcholine Lipids. The Journal of Physical Chemistry B 2012, 116 (10), 3164-3179. 14. Klauda, J. B.; Venable, R. M.; Freites, J. A.; O'Connor, J. W.; Tobias, D. J.; Mondragon-Ramirez, C.; Vorobyov, I.; MacKerell, A. D.; Pastor, R. W., Update of the CHARMM All-Atom Additive Force Field for Lipids: Validation on Six Lipid Types. Journal of Physical Chemistry B 2010, 114 (23), 7830-7843. 15. Dickson, C. J.; Madej, B. D.; Skjevik, Å. A.; Betz, R. M.; Teigen, K.; Gould, I. R.; Walker, R. C., Lipid14: The Amber Lipid Force Field. Journal of Chemical Theory and Computation 2014, 10 (2), 865-879. 16. Chiu, S.-W.; A. Pandit, S.; L. Scott, H.; Jakobsson, E., An Improved United Atom Force Field for Simulation of Mixed Lipid Bilayers. The Journal of Physical Chemistry B 2009, 113 (9), 2748-2763.

17. Leonard, A. N.; Wang, E.; Monje-Galvan, V.; Klauda, J. B., Developing and Testing of Lipid Force Fields with Applications to Modeling Cellular Membranes. Chemical Reviews 2019, 119 (9), 6227-6269. 
18. Klauda, J. B.; Monje, V.; Kim, T.; Im, W., Improving the CHARMM force field for polyunsaturated fatty acid chains. Journal of Physical Chemistry B 2012, 116 (31), 9424-9431.

19. Venable, R. M.; Sodt, A. J.; Rogaski, B.; Rui, H.; Hatcher, E.; MacKerell, A. D.; Pastor, R. W.;

Klauda, J. B., CHARMM all-atom additive force field for sphingomyelin: Elucidation of hydrogen bonding and of positive curvature. Biophysical Journal 2014, 107 (1), 134-145.

20. Venable, R. M.; Luo, Y.; Gawrisch, K.; Roux, B.; Pastor, R. W., Simulations of anionic lipid membranes: Development of interaction-specific ion parameters and validation using NMR data. Journal of Physical Chemistry B 2013, 117 (35), 10183-10192.

21. West, A.; Zoni, V.; Teague, W. E.; Leonard, A. N.; Vanni, S.; Gawrisch, K.; Tristram-Nagle, S.; Sachs, J. N.; Klauda, J. B., How Do Ethanolamine Plasmalogens Contribute to Order and Structure of Neurological Membranes? The Journal of Physical Chemistry B 2020, 124 (5), 828-839.

22. Leonard, A. N.; Pastor, R. W.; Klauda, J. B., Parameterization of the CHARMM All-Atom Force Field for Ether Lipids and Model Linear Ethers. Journal of Physical Chemistry B 2018.

23. Wu, Emilia L.; Engström, O.; Jo, S.; Stuhlsatz, D.; Yeom, Min S.; Klauda, Jeffery B.; Widmalm, G.; Im, W., Biophysical Journal 2013, 105 (6), 1444-1455.

24. Wang, E.; Klauda, J. B., Molecular Dynamics Simulations of Ceramide and CeramidePhosphatidylcholine Bilayers. The Journal of Physical Chemistry B 2017, 121 (43), 10091-10104.

25. Venable, R. M.; Brown, F. L. H.; Pastor, R. W., Mechanical Properties of Lipid Bilayers from Molecular Dynamics Simulation. Chemistry and Physics of Lipids 2015, 192, 60-74.

26. Klauda, J. B.; Wu, X.; Pastor, R. W.; Brooks, B. R., Long-Range Lennard-Jones and Electrostatic Interactions in Interfaces: Application of the Isotropic Periodic Sum Method. The Journal of Physical Chemistry B 2007, 111 (17), 4393-4400.

27. Leonard, A. N.; Simmonett, A. C.; Pickard, F. C.; Huang, J.; Venable, R. M.; Klauda, J. B.; Brooks, B. R.; Pastor, R. W., Comparison of Additive and Polarizable Models with Explicit Treatment of Long-Range Lennard-Jones Interactions Using Alkane Simulations. Journal of Chemical Theory and Computation 2018, 14 (2), 948-958.

28. Mecke, M.; Winkelmann, J.; Fischer, J., Molecular Dynamics Simulation of the Liquid-Vapor Interface: The Lennard-Jones Fluid. Journal of Chemical Physics 1997, 107, 9264-9270.

29. Krämer, A.; C. Pickard, F.; Huang, J.; M. Venable, R.; C. Simmonett, A.; Reith, D.; N. Kirschner, K.; W. Pastor, R.; R. Brooks, B., Interactions of Water and Alkanes: Modifying Additive Force Fields to Account for Polarization Effects. Journal of Chemical Theory and Computation 2019, 15 (6), 3854-3867. 30. Schlenkrich, M.; Brickmann, J.; MacKerell, A. D.; Karplus, M., An Empirical Potential Energy Function for Phospholipids: Criteria for Parameter Optimization and Applications. In Biological Membranes: A 
Molecular Perspective from Computation and Experiment, Merz, K. M.; Roux, B., Eds. Birkhäuser Boston: Boston, MA, 1996; pp 31-81.

31. Pastor, R. W.; MacKerell, A. D., Development of the CHARMM Force Field for Lipids. The Journal of Physical Chemistry Letters 2011, 2 (13), 1526-1532.

32. Venable, R. M.; Zhang, Y.; Hardy, B. J.; Pastor, R. W., Molecular dynamics simulations of a lipid bilayer and of hexadecane: An investigation of membrane fluidity. Science 1993, 262, 223-226.

33. Tu, K.; Tobias, D. J.; Blasie, J. K.; Klein, M. L., Molecular dynamics investigation of the structure of a fully hydrated gel-phase dipalmitoylphosphatidylcholine bilayer. Biophysical Journal 1996, 70 (2), 595-608.

34. Tieleman, D. P.; Marrink, S. J.; Berendsen, H. J. C., A computer perspective of membranes: molecular dynamics studies of lipid bilayer systems. Biochimica et Biophysica Acta (BBA) - Reviews on Biomembranes 1997, 1331 (3), 235-270.

35. Abraham, M. J.; Murtola, T.; Schulz, R.; Páll, S.; Smith, J. C.; Hess, B.; Lindahl, E., GROMACS: High performance molecular simulations through multi-level parallelism from laptops to supercomputers. SoftwareX 2015, 1-2, 19-25.

36. Brooks, B. R.; Brooks, C. L.; Mackerell, A. D.; Nilsson, L.; Petrella, R. J.; Roux, B.; Won, Y.; Archontis, G.; Bartels, C.; Boresch, S.; Caflisch, A.; Caves, L.; Cui, Q.; Dinner, A. R.; Feig, M.; Fischer, S.; Gao, J.; Hodoscek, M.; Im, W.; Kuczera, K.; Lazaridis, T.; Ma, J.; Ovchinnikov, V.; Paci, E.; Pastor, R. W.; Post, C. B.; Pu, J. Z.; Schaefer, M.; Tidor, B.; Venable, R. M.; Woodcock, H. L.; Wu, X.; Yang, W.; York, D. M.; Karplus, M., CHARMM: The biomolecular simulation program. Journal of Computational Chemistry 2009, 30 (10), 1545-1614.

37. Salomon-Ferrer, R.; Case, D. A.; Walker, R. C., An overview of the Amber biomolecular simulation package. WIREs Computational Molecular Science 2013, 3 (2), 198-210.

38. Eastman, P.; Swails, J.; Chodera, J. D.; McGibbon, R. T.; Zhao, Y.; Beauchamp, K. A.; Wang, L.-P.; Simmonett, A. C.; Harrigan, M. P.; Stern, C. D.; Wiewiora, R. P.; Brooks, B. R.; Pande, V. S., OpenMM 7: Rapid development of high performance algorithms for molecular dynamics. PLOS Computational Biology 2017, 13 (7), e1005659.

39. Edholm, O., Chapter 3 Time and Length Scales in Lipid Bilayer Simulations. 2008.

40. Zhuang, X.; Makover, J. R.; Im, W.; Klauda, J. B., A Systematic Molecular Dynamics Simulation Study of Temperature Dependent Bilayer Structural Properties. Biochimica et Biophysica Acta (BBA) -

Biomembranes 2014, 1838 (10), 2520-2529.

41. Zhuang, X.; Dávila-Contreras, E. M.; Beaven, A. H.; Im, W.; Klauda, J. B., An extensive simulation study of lipid bilayer properties with different head groups, acyl chain lengths, and chain saturations.

Biochimica et Biophysica Acta - Biomembranes 2016, 1858 (12), 3093-3104. 
42. Krämer, A.; Hülsmann, M.; Köddermann, T.; Reith, D., Automated parameterization of intermolecular pair potentials using global optimization techniques. Computer Physics Communications 2014, 185 (12), 32283239.

43. Hülsmann, M.; Kirschner, K. N.; Krämer, A.; Heinrich, D. D.; Krämer-Fuhrmann, O.; Reith, D., Optimizing Molecular Models Through Force-Field Parameterization via the Efficient Combination of Modular Program Packages. In Foundations of Molecular Modeling and Simulation: Select Papers from FOMMS 2015, Snurr, R. Q.; Adjiman, C. S.; Kofke, D. A., Eds. Springer Singapore: Singapore, 2016; pp 53-77.

44. Wang, L.-P.; J. Martinez, T.; S. Pande, V., Building Force Fields: An Automatic, Systematic, and Reproducible Approach. The Journal of Physical Chemistry Letters 2014, 5 (11), 1885-1891.

45. Shirts, M. R.; Chodera, J. D., Statistically optimal analysis of samples from multiple equilibrium states. The Journal of Chemical Physics 2008, 129 (12), 124105.

46. Messerly, R. A.; Razavi, S. M.; Shirts, M. R., Configuration-Sampling-Based Surrogate Models for Rapid Parameterization of Non-Bonded Interactions. Journal of Chemical Theory and Computation 2018, 14 (6), 3144-3162.

47. Reith, D.; Meyer, H.; Müller-Plathe, F., CG-OPT: A software package for automatic force field design. Computer Physics Communications 2002, 148 (3), 299-313.

48. Bourasseau, E.; Haboudou, M.; Boutin, A.; Fuchs, A. H.; Ungerer, P., New optimization method for intermolecular potentials: Optimization of a new anisotropic united atoms potential for olefins: Prediction of equilibrium properties. The Journal of Chemical Physics 2003, 118 (7), 3020-3034.

49. Hülsmann, M.; Köddermann, T.; Vrabec, J.; Reith, D., GROW: A gradient-based optimization workflow for the automated development of molecular models. Computer Physics Communications 2010, 181 (3), 499-513.

50. Mobley, D. L.; Bannan, C. C.; Rizzi, A.; Bayly, C. I.; Chodera, J. D.; Lim, V. T.; Lim, N. M.; Beauchamp, K. A.; Slochower, D. R.; Shirts, M. R.; Gilson, M. K.; Eastman, P. K., Escaping Atom Types in Force Fields Using Direct Chemical Perception. Journal of Chemical Theory and Computation 2018, 14 (11), 6076-6092.

51. Qiu, Y. S., Daniel; Boothroyd, Simon; Jang, Hyesu; Wagner, Jeffrey; Bannan, Caitlin C.; et al.. Development and Benchmarking of Open Force Field v1.0.0, the Parsley Small Molecule Force Field. ChemRxiv 2020, Preprint.

52. McKiernan, K. A.; Wang, L.-P.; Pande, V. S., Training and Validation of a Liquid-Crystalline Phospholipid Bilayer Force Field. Journal of Chemical Theory and Computation 2016, 12 (12), 5960-5967. 53. Yu, Y.; Klauda, J. B., Update of the CHARMM36 United Atom Chain Model for Hydrocarbons and Phospholipids. The Journal of Physical Chemistry B 2020. 
54. Wennberg, C. L.; Murtola, T.; Páll, S.; Abraham, M. J.; Hess, B.; Lindahl, E., Direct-Space

Corrections Enable Fast and Accurate Lorentz-Berthelot Combination Rule Lennard-Jones Lattice Summation. Journal of Chemical Theory and Computation 2015, 11 (12), 5737-5746.

55. Zwanzig, R. W., High-Temperature Equation of State by a Perturbation Method. I. Nonpolar Gases. The Journal of Chemical Physics 1954, 22 (8), 1420-1426.

56. Oliphant, T. E., Python for scientific computing. Computing in Science and Engineering 2007.

57. Foglia, F.; Lawrence, M. J.; Lorenz, C. D.; McLain, S. E., On the hydration of the phosphocholine headgroup in aqueous solution. The Journal of Chemical Physics 2010, 133 (14), 145103.

58. Rhys, N. H.; Duffy, I. B.; Sowden, C. L.; Lorenz, C. D.; McLain, S. E., On the hydration of DOPE in solution. The Journal of Chemical Physics 2019, 150 (11), 115104.

59. Kučerka, N.; Nieh, M. P.; Katsaras, J., Fluid Phase Lipid Areas and Bilayer Thicknesses of Commonly Used Phosphatidylcholines as a Function of Temperature. Biochimica et Biophysica Acta Biomembranes 2011, 1808 (11), 2761-2771.

60. Seelig, A.; Seelig, J., Dynamic structure of fatty acyl chains in a phospholipid bilayer measured by deuterium magnetic resonance. Biochemistry 1974, 13 (23), 4839-4845.

61. Seelig, A.; Seelig, J., Bilayers of Dipalmitoyl-3-sn-

Phosphatidylcholine: Conformational Differences between the Fatty Acyl Chains. BBA - Biomembranes 1975, $406(1), 1-5$.

62. Klauda, J. B.; Eldho, N. V.; Gawrisch, K.; Brooks, B. R.; Pastor, R. W., Collective and Noncollective Models of NMR Relaxation in Lipid Vesicles and Multilayers. Journal of Physical Chemistry B 2008, 112 (19), 5924-5929.

63. Kučerka, N.; Liu, Y.; Chu, N.; Petrache, H. I.; Tristram-Nagle, S.; Nagle, J. F., Structure of fully hydrated fluid phase DMPC and DLPC lipid bilayers using x-ray scattering from oriented multilamellar arrays and from unilamellar vesicles. Biophysical Journal 2005, 88 (4), 2626-2637.

64. Somerharju, P. J.; Virtanen, J. A.; Eklund, K. K.; Vainio, P.; Kinnunen, P. K. J., 1-Palmitoyl-2Pyrenedecanoyl Glycerophospholipids as Membrane Probes: Evidence for Regular Distribution in LiquidCrystalline Phosphatidylcholine Bilayers. Biochemistry 1985, 24 (11), 2773-2781.

65. Nagle, J. F.; Tristram-Nagle, S., Structure of Lipid Bilayers. Biochimica et Biophysica Acta - Reviews on Biomembranes 2000, 1469 (3), 159-195.

66. Botan, A.; Favela-Rosales, F.; Fuchs, P. F. J.; Javanainen, M.; Kanduč, M.; Kulig, W.; Lamberg, A.; Loison, C.; Lyubartsev, A.; Miettinen, M. S.; Monticelli, L.; Määttä, J.; Ollila, O. H. S.; Retegan, M.; Róg, T.; Santuz, H.; Tynkkynen, J., Toward Atomistic Resolution Structure of Phosphatidylcholine Headgroup and 
Glycerol Backbone at Different Ambient Conditions. The Journal of Physical Chemistry B 2015, 119 (49), 15075-15088.

67. Guvench, O.; MacKerell, A. D., Automated conformational energy fitting for force-field development. Journal of Molecular Modeling 2008, 14 (8), 667-679.

68. Jo, S.; Kim, T.; Iyer, V. G.; Im, W., CHARMM-GUI: A Web-Based Graphical User Interface for CHARMM. Journal of Computational Chemistry 2008, 29 (11), 1859-1865.

69. Jo, S.; Lim, J. B.; Klauda, J. B.; Im, W., CHARMM-GUI Membrane Builder for Mixed Bilayers and Its Application to Yeast Membranes. Biophysical Journal 2009, 97 (1), $50-58$.

70. Wu, E. L.; Cheng, X.; Jo, S.; Rui, H.; Song, K. C.; Dávila-Contreras, E. M.; Qi, Y.; Lee, J.; MonjeGalvan, V.; Venable, R. M.; Klauda, J. B.; Im, W., CHARMM-GUI Membrane Builder toward realistic biological membrane simulations. Journal of Computational Chemistry 2014, 35 (27), 1997-2004.

71. Wu, X.; Brooks, B. R., Self-guided Langevin dynamics simulation method. Chemical Physics Letters 2003, 381 (3), 512-518.

72. Durell, S. R.; Brooks, B. R.; Ben-Naim, A., Solvent-induced forces between two hydrophilic groups. Journal of Physical Chemistry 1994, 98 (8), 2198-2202.

73. Jorgensen, W. L.; Chandrasekhar, J.; Madura, J. D.; Impey, R. W.; Klein, M. L., Comparison of simple potential functions for simulating liquid water. The Journal of Chemical Physics 1983, 79 (2), $926-935$.

74. Hoover, W. G., Canonical dynamics: Equilibrium phase-space distributions. Physical Review A 1985, 31 (3), 1695-1697.

75. Nosé, S.; Klein, M. L., Constant pressure molecular dynamics for molecular systems. Molecular Physics 1983, 50 (5), 1055-1076.

76. Andersen, H. C., Molecular dynamics simulations at constant pressure and/or temperature. The Journal of Chemical Physics 1980, 72 (4), 2384-2393.

77. McGibbon, Robert T.; Beauchamp, Kyle A.; Harrigan, Matthew P.; Klein, C.; Swails, Jason M.; Hernández, Carlos X.; Schwantes, Christian R.; Wang, L.-P.; Lane, Thomas J.; Pande, Vijay S., MDTraj: A Modern Open Library for the Analysis of Molecular Dynamics Trajectories. Biophysical Journal 2015, 109 (8), 1528-1532.

78. Oliphant, T. E., Python for Scientific Computing. Computing in Science \& Engineering 2007, 9 (3), $10-$ 20.

79. Phillips, J. C.; Braun, R.; Wang, W.; Gumbart, J.; Tajkhorshid, E.; Villa, E.; Chipot, C.; Skeel, R. D.; Kalé, L.; Schulten, K., Scalable molecular dynamics with NAMD. Journal of Computational Chemistry 2005, 26 (16), 1781-1802.

80. Nagle, J. F., Area/lipid of bilayers from NMR. Biophysical Journal 1993, 64 (5), 1476-1481. 
81. Gross, J. D.; Warschawski, D. E.; Griffin, R. G., Dipolar Recoupling in MAS NMR: A Probe for Segmental Order in Lipid Bilayers. Journal of the American Chemical Society 1997, 119 (4), 796-802.

82. Hong, M.; Schmidt-Rohr, K.; Pines, A., NMR Measurement of Signs and Magnitudes of C-H Dipolar Couplings in Lecithin. Journal of the American Chemical Society 1995, 117 (11), 3310-3311.

83. Hilgemann, D. W., Local PIP2 signals: when, where, and how? Pflügers Archiv - European Journal of Physiology 2007, 455 (1), 55-67.

84. van den Bogaart, G.; Meyenberg, K.; Risselada, H. J.; Amin, H.; Willig, K. I.; Hubrich, B. E.; Dier, M.; Hell, S. W.; Grubmüller, H.; Diederichsen, U.; Jahn, R., Membrane protein sequestering by ionic proteinlipid interactions. Nature 2011, 479 (7374), 552-555.

85. Toner, M.; Vaio, G.; McLaughlin, A.; McLaughlin, S., Adsorption of cations to phosphatidylinositol 4,5-bisphosphate. Biochemistry 1988, 27 (19), 7435-7443.

86. Jiang, Z.; Redfern, R. E.; Isler, Y.; Ross, A. H.; Gericke, A., Cholesterol stabilizes fluid phosphoinositide domains. Chemistry and Physics of Lipids 2014, 182, 52-61.

87. Wang, Y.-H.; Collins, A.; Guo, L.; Smith-Dupont, K. B.; Gai, F.; Svitkina, T.; Janmey, P. A., Divalent Cation-Induced Cluster Formation by Polyphosphoinositides in Model Membranes. Journal of the American Chemical Society 2012, 134 (7), 3387-3395.

88. Levental, I.; Janmey, P. A.; Cēbers, A., Electrostatic Contribution to the Surface Pressure of Charged Monolayers Containing Polyphosphoinositides. Biophysical Journal 2008, 95 (3), 1199-1205.

89. Alvares, D. S.; dos Santos Cabrera, M. P.; Ruggiero Neto, J., Chapter Two - Strategies for Exploring Electrostatic and Nonelectrostatic Contributions to the Interaction of Helical Antimicrobial Peptides with Model Membranes. In Advances in Biomembranes and Lipid Self-Assembly, Iglič, A.; Kulkarni, C. V.; Rappolt, M., Eds. Academic Press: 2016; Vol. 24, pp 43-73.

90. Petelska, A. D.; Naumowicz, M.; Figaszewski, Z. A., The Influence of pH on Phosphatidylethanolamine Monolayer at the Air/Aqueous Solution Interface. Cell Biochemistry and Biophysics 2013, 65 (2), 229-235.

91. Cseh, R.; Benz, R., The Adsorption of Phloretin to Lipid Monolayers and Bilayers Cannot Be Explained by Langmuir Adsorption Isotherms Alone. Biophysical Journal 1998, 74 (3), 1399-1408.

92. Gawrisch, K.; Parsegian, V. A.; Hajduk, D. A.; Tate, M. W.; Gruner, S. M.; Fuller, N. L.; Rand, R. P., Energetics of a hexagonal-lamellar-hexagonal-phase transition sequence in dioleoylphosphatidylethanolamine membranes. Biochemistry 1992, 31 (11), 2856-2864. 
TOC Graphic:

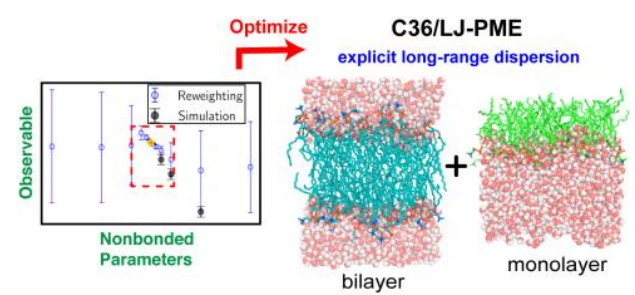

